\title{
Accumulation of Neurofascin at Nodes of Ranvier Is Regulated by a Paranodal Switch
}

\author{
Yanqing Zhang, ${ }^{1 *}$ Stephanie Yuen, ${ }^{1}{ }^{\circledR}$ Elior Peles, ${ }^{3}$ and James L. Salzer ${ }^{1,2 *}$ \\ ${ }^{1}$ Neuroscience Institute and Departments of Neuroscience and Physiology and, ${ }^{2}$ Neurology, New York University School of Medicine, New York, \\ New York 10016, and ${ }^{3}$ Department of Molecular Cell Biology, Weizmann Institute of Science, Rehovot, 76100, Israel
}

The paranodal junctions flank mature nodes of Ranvier and provide a barrier between ion channels at the nodes and juxtaparanodes. These junctions also promote node assembly and maintenance by mechanisms that are poorly understood. Here, we examine their role in the accumulation of NF186, a key adhesion molecule of PNS and CNS nodes. We previously showed that NF186 is initially targeted/accumulates via its ectodomain to forming PNS (hemi)nodes by diffusion trapping, whereas it is later targeted to mature nodes by a transport-dependent mechanism mediated by its cytoplasmic segment. To address the role of the paranodes in this switch, we compared accumulation of NF186 ectodomain and cytoplasmic domain constructs in WT versus paranode defective (i.e., Caspr-null) mice. Both pathways are affected in the paranodal mutants. In the PNS of Caspr-null mice, diffusion trapping mediated by the NF186 ectodomain aberrantly persists into adulthood, whereas the cytoplasmic domain/transport-dependent targeting is impaired. In contrast, accumulation of NF186 at CNS nodes does not undergo a switch; it is predominantly targeted to both forming and mature CNS nodes via its cytoplasmic domain and requires intact paranodes. Fluorescence recovery after photobleaching analysis indicates that the paranodes provide a membrane diffusion barrier that normally precludes diffusion of NF186 to nodes. Linkage of paranodal proteins to the underlying cytoskeleton likely contributes to this diffusion barrier based on $4.1 \mathrm{~B}$ and $\beta \mathrm{II}$ spectrin expression in Caspr-null mice. Together, these results implicate the paranodes as membrane diffusion barriers that regulate targeting to nodes and highlight differences in the assembly of PNS and CNS nodes.

Key words: myelin; neurofascin; node of Ranvier; oligodendrocyte; paranodes; Schwann cell

Significance Statement

Nodes of Ranvier are essential for effective saltatory conduction along myelinated axons. A major question is how the various axonal proteins that comprise the multimeric nodal complex accumulate at this site. Here we examine how targeting of NF186, a key nodal adhesion molecule, is regulated by the flanking paranodal junctions. We show that the transition from diffusion-trapping to transport-dependent accumulation of NF186 requires the paranodal junctions. We also demonstrate that these junctions are a barrier to diffusion of axonal proteins into the node and highlight differences in PNS and CNS node assembly. These results provide new insights into the mechanism of node assembly and the pathophysiology of neurologic disorders in which impaired paranodal function contributes to clinical disability.

\section{Introduction}

Nodes of Ranvier are the sites of action potential regeneration along myelinated axons. They are enormously enriched in voltage-gated sodium channels that are part of a multimeric complex

Received Apr. 10, 2019; revised Apr. 29, 2020; accepted June 2, 2020.

Author contributions: Y.Z. and J.L.S. designed research; Y.Z. and S.Y. performed research; Y.Z., E.P., and J.L.S. analyzed data; Y.Z. wrote the first draft of the paper; Y.Z., E.P., and J.L.S. edited the paper; J.L.S. wrote the paper.

This work was supported by National Institutes of Health Grant NS043474 to J.L.S. We thank M. Rasband, M. Bhat, and S. Lux for kindly providing antibodies.

The authors declare no competing financial interests.

Correspondence should be addressed to Yanqing Zhang at Yanqing.Zhang@nyulangone.org or James L. Salzer at James.Salzer@nyulangone.org.

https://doi.org/10.1523/JNEUROSCI.0830-19.2020

Copyright $\odot 2020$ the authors that also includes the cell adhesion molecules (CAMs) neurofascin 186 (NF186) and NrCAM, and a cytoskeletal scaffold of ankyrin $\mathrm{G}$ and $\beta$ IV spectrin. This scaffold is, in turn, linked to actin rings (D'Este et al., 2017) and a contractile myosin cytoskeleton (Berger et al., 2018).

A key question is what drives assembly of this protein complex. Important insights have emerged from the sequence of events during node assembly and maturation. During development, sodium channels initially cluster as hemi-nodes, that is, sodium channel complexes adjacent to individual myelin sheaths (Vabnick et al., 1996; Ching et al., 1999). Two adjacent heminodes are believed to coalesce to form each mature node, which are spatially delineated by the flanking paranodal junctions (PNJs) (Rasband, 2013). The PNJs themselves form between the lateral edges at the ends of myelinating glia (which appear as paranodal 
loops in electron microscopy cross-section) and the closely apposed axolemma (Salzer, 2003). By electron microscopy, the junctions have the appearance of regularly spaced transverse bands that extend between the axon and glial membranes. These transverse bands likely correspond to a cis-complex of Caspr and contactin on the axon, which interacts in trans with NF155 on the apposed glial (i.e., Schwann cell or oligodendrocyte) membrane (Bhat et al., 2001; Boyle et al., 2001; Charles et al., 2002; Sherman et al., 2005). The cytoplasmic domain of Caspr binds to $4.1 \mathrm{~B}$, which functions as an adaptor linking this complex to the underlying $\alpha \mathrm{II} / \beta$ II spectrin complex (Ogawa et al., 2006), which in turn binds to actin creating a stable and flexible actin-spectrin cytoskeletal network (Bennett and Lorenzo, 2013).

The initial event of PNS node formation is the accumulation of NF186 at heminodes (Lambert et al., 1997; Lustig et al., 2001). NF186 is recruited to these early nodes from a preexisting pool on the axon surface (Y. Zhang et al., 2012) by diffusion trapping that results from interactions of its ectodomain (Dzhashiashvili et al., 2007) with a cognate receptor complex on the Schwann cell microvilli (i.e., gliomedin and NrCAM) (Eshed et al., 2005; Feinberg et al., 2010). NF186 then recruits ankyrin G and thereby ion channels to the site (Eshed et al., 2005; Sherman et al., 2005; Dzhashiashvili et al., 2007). A second mechanism that subsequently contributes to, and is sufficient by itself to drive node assembly, is mediated by the flanking PNJs. Thus, in NF186 KO mice, heminode formation is disrupted, whereas the $\mathrm{Na}_{\mathrm{v}} / \mathrm{AnkG}$ complex still accumulates at mature nodes in a paranode-dependent manner (Feinberg et al., 2010).

CNS nodes have been proposed to assemble similarly to PNS nodes (Susuki et al., 2013), although there are important differences. In the PNS, node formation and NF186 and NaV accumulation precede paranode formation (Melendez-Vasquez et al., 2001). In the CNS, the PNJs form first, followed by deposition of the nodal cytoskeleton, then $\mathrm{NaV}$, and finally NF186, which is recruited with a delay (Rasband et al., 1999; Melendez-Vasquez et al., 2001; Jenkins and Bennett, 2002). This lag in NF186 accumulation may reflect delayed expression of NF186 ligands at CNS nodes. Further, CNS nodes are not contacted by microvilli and lack gliomedin. Instead, NF186 interacts with and may be stabilized by extracellular matrix components (ECMs), which accumulate with a delay after CNS nodes form (Oohashi et al., 2002; Dours-Zimmermann et al., 2009; Susuki et al., 2013). In addition, soluble factors released by oligodendendrocytes have been implicated in early clustering of the nodal complex before myelination in a subset of CNS neurons (Kaplan et al., 1997, 2001; Freeman et al., 2015; Dubessy et al., 2019). Together, these findings suggest important differences in PNS and CNS node assembly; they also suggest CNS nodes may rely more on paranode-dependent rather than NF186-directed clustering mechanisms. In potential agreement, the glial form of neurofascin (NF155) rescues sodium clustering in neurofascin ${ }^{-1-}$ nodes more efficiently in the CNS than in the PNS (Sherman et al., 2005; Zonta et al., 2008; Amor et al., 2017).

The mechanisms by which paranodes contribute to assembly of PNS and CNS nodes are not known. This role of the PNJs in node assembly and maintenance is of clinical significance as the paranodes are increasingly recognized as a site of pathology in neuropathies and demyelinating disorders (Faivre-Sarrailh and Devaux, 2013; Manso et al., 2019; Faivre-Sarrailh, 2020). The PNJs have long been considered to function as barriers between the voltage-gated sodium channels at the node and of potassium channels at the juxtaparanodes (Rosenbluth, 2009). By providing a membrane barrier for these transmembrane proteins, the paranodes may restrict and concentrate sodium channels as two paranodes progressively approach each other during myelination (Pedraza et al., 2001; Feinberg et al., 2010). An alternative role of the paranodes is suggested by studies of how NF186 is targeted to PNS nodes. In particular, we reported that NF186 targeting/ accumulation at nodes switches from initial ectodomain-dependent accumulation at forming nodes to a cytoplasmic domain-dependent clustering at mature nodes, which are flanked by PNJs (Y. Zhang et al., 2012). These results suggested the PNJs may be responsible for the switch in the targeting of NF186 at nodes from an initial diffusion-trapping mechanism to a subsequent transport-dependent mechanism.

Here, we examine directly the role of paranodes in node formation and this switch in NF186 accumulation. We used paranode-defective, Caspr null mice to analyze targeting of NF186 constructs to both PNS and CNS nodes. Our results show that the PNJs function as barriers to the dynamic diffusion of membrane proteins, which thereby block ectodomain-dependent NF186 diffusion trapping and favor cytoplasmic-domain mediated accumulation of NF186 at nodes. We also show that clustering of NF186 at CNS nodes, unlike PNS nodes, principally relies on its cytoplasmic domain and that this accumulation is abrogated in the Caspr nulls. Together, these results implicate the paranodes as key regulators of the accumulation of components at nodes and highlight important differences in the assembly of PNS and CNS nodes.

\section{Material and Methods}

Tissue culture. Caspr KO DRG neurons were obtained from breeding of Cntnap1-null mice (Feinberg et al., 2010). Cocultures of Schwann cells and mouse DRG neurons were established as described previously (Y. Zhang et al., 2012). Briefly, DRGs were removed from E13.5 mouse embryos, dissociated with $0.25 \%$ trypsin, and plated onto Matrigel (BD Bioscience)-coated coverslips. After cycling with antimitotics to eliminate non-neuronal cells, Schwann cells were added to the cultures and maintained in $\mathrm{C}$ media (containing $10 \% \mathrm{FBS}, 50 \mathrm{ng} / \mathrm{ml} 2.5 \mathrm{~S}$ nerve growth factor, $0.4 \%$ glucose, and $20 \mathrm{~mm}$ L-glutamine in minimum essential medium) for $1-3 \mathrm{~d}$ before adding $50 \mu \mathrm{g} / \mathrm{ml}$ ascorbic acid to allow myelination to ensue. For experiments with mature cocultures, cultures were maintained in myelinating condition for 4-6 weeks before experiments were conducted. Expression of inducible NF186 and mutant constructs was induced by adding $1 \mu \mathrm{g} / \mathrm{ml}$ doxycycline to the culture media; cultures were fixed and analyzed 1 week after induction.

cDNA constructs and viral production. NF186 and chimeric constructs were subcloned into the pSLIK vector, and viruses were generated as described previously (Y. Zhang et al., 2012). MyrGFP was generated by inserting the sequence 5 '-GGGAG TAGCAAGAGCAAGCCTAAGGACCCCAGCCAGCGCCGGC GC-3' into FUGW viral vector through BamHI/AgeI sites. Human Caspr was tagged at its C-terminus with mKate2, a monomeric form of red fluorescence protein (gift of E. Siggia), and this construct was inserted into the FUGW lentival vector.

Immunofluorescence and antibodies. Neuron and myelinating cocultures were fixed in $2 \%-4 \%$ PFA for $10 \mathrm{~min}$, permeabilized with $100 \%$ methanol at $-20^{\circ} \mathrm{C}$ for $20 \mathrm{~min}$, blocked with buffer containing $1 \%$ donkey serum, $5 \%$ BSA, and $0.2 \%$ Triton $\mathrm{X}-100$ in $1 \times$ PBS for $30 \mathrm{~min}$, and stained with primary and secondary antibodies. Sciatic nerves and optic nerves were dissected and fixed in 1\%-4\% PFA for 1-2 h. After washing in cold PBS, the sciatic nerves were teased and transferred to slides, and then 
stored at $-80^{\circ} \mathrm{C}$ until use; optic nerves were submerged in $30 \%$ sucrose overnight and then embedded in Tissue-Tek and stored at $-80^{\circ} \mathrm{C}$ until use. To prepare samples for Triton extraction, sciatic nerves were processed without fixation, frozen in a dry ice/ ethanol bath, and embedded in Tissue-Tek OCT and then cut as $10 \mu \mathrm{m}$ cryostat sections.

Antibodies used include mouse anti-ankyrin G (1:200; Antibodies Incorporated, catalog \#75-146, RRID:AB_10673030), rabbit anti-ankyrin G (1:2000; Thermo Fisher Scientific, catalog \#A-11122, RRID:AB_221569), goat anti-GFP (1:2000; Bio-Rad, catalog \#AHP975, RRID:AB_566990), chicken anti-MBP (1:100; Millipore, catalog \#AB9348, RRID:AB_2140366), chicken and rabbit anti- $\beta$ IV spectrin (1:2000; M. Rasband, Baylor College of Medicine, Houston, rabbit antibody RRID:AB_2315634, chicken antibody RRID:AB_2827639), and guinea-pig anti-Caspr (1:2000; M. Bhat, University of Texas, San Antonio, RRID:AB_2827640), mouse anti- $\beta$ II spectrin (1:400, BD Biosciences, catalog \#612562, RRID:AB_399853), and rabbit 4.1B (1:1000, Elior Peles, Weizmann Institute of Science, Rehovot, Israel, RRID:AB_2827725). Secondary donkey antibodies conjugated to AlexaFluor-488, -568, -647 were obtained from Jackson ImmunoResearch Laboratories and used at 1:200-1:500 dilution.

Preparation of nerve lysates and immunoblotting. Sciatic and optic nerves were lysed in a solution containing inhibitor mixture (Sigma-Aldrich, catalog \#05892970001), 1\% SDS, $5 \mathrm{mM}$ EDTA, $1 \%$ NP-40, 1\% sodium deoxycholate, $1 \%$ Triton X-100 in $50 \mathrm{mM}$ Tris, $\mathrm{pH}$ 7.5. Protein concentrations were determined by the Pierce BCA protein assay kit (Thermo Fisher Scientific, cata$\log \# 23227)$; protein samples $(20-40 \mu \mathrm{g})$ were fractionated by $8 \%$ SDS-PAGE and blotted onto nitrocellulose blotting membrane (GE Healthcare Life Sciences, catalog \#10600033). Appropriate regions of the blot were cut out and incubated with specific primary and secondary antibodies. Blots were developed using LI-COR Biosciences Odyssey Fc Imaging System. Primary antibodies used include chicken anti-Neurofascin (1:4000; R\&D Systems, catalog \#AF3235, RRID:AB_10890736), rabbit antiankyrin G (1:2000; Thermo Fisher Scientific, catalog \#A-11122, RRID:AB_221569), mouse anti- $\beta$ II spectrin (1:3000, BD Biosciences, catalog \#612562, RRID:AB_399853), and mouse anti- $\alpha$-tubulin (1;3000, Sigma Millipore, catalog \#T9026, RRID: AB_477593). Secondary antibodies with IRDye 800CW and 680RD were obtained from LI-COR Biosciences.

Triton X-100 extraction. Nonfixed sciatic nerve sections or cultures were incubated in extraction buffer (30 mM PIPES, 1 $\mathrm{mM} \mathrm{MgCl} 2,5 \mathrm{mM}$ EDTA, $0.5 \%$ Triton X-100) for $30 \mathrm{~min}$ (tissue sections) or $10 \mathrm{~min}$ (cultures) at $37^{\circ} \mathrm{C}$, and then rinsed in $\mathrm{PBS}$, fixed in 4\% PFA, and processed for immunofluorescence.

Imaging and quantification. All images for cultures and tissue slides are confocal images taken with an LSM 510 Meta, LSM 710 , or LSM 880 confocal microscope (Carl Zeiss). Quantitation was done with ImageJ. To determine the intensity distribution of transgenes at the node, raw intensity measurements were converted to relative intensity. In particular, the highest nodal intensity in WT versus Caspr KO nerve pairs was set as 100; all other nodal intensities were scaled proportionately accordingly. Results from three or four comparable pairs were pooled. Statistical analysis was done with Prism software using unpaired $t$ test.

Fluorescence recovery after photobleaching (FRAP) analysis. DRG neurons from WT and Caspr KO mice were plated onto single-well, $35 \mathrm{~mm}$ MatTek cell culture dishes. Cultures were then infected with lentivirus encoding ICAM1-EGFP or MyrGFP, then further infected with hCaspr-mkate2 in the case of WT neurons or NF186-mCherry in the case of Caspr KO neurons.
Myelinating cocultures were prepared as described above. Cultures were pretreated with $66 \mathrm{nM}$ nocodazole (Sigma Millipore), a microtubule-disrupting agent, to block axonal transport beginning $4 \mathrm{~h}$ before photobleaching to prevent vesicular transport that might confound analysis. Cultures were maintained in phenol red-free $\mathrm{C}$ media buffered with $10 \mathrm{mM}$ HEPES at $37^{\circ} \mathrm{C}$ during the experiment. FRAP experiments were conducted with an LSM 710 or 880 microscope (Carl Zeiss) with the $63 \times$ or $100 \times$ oil immersion objective; FRAP analysis was performed as previously reported (Snapp et al., 2003). The diffusion coefficient was determined using an inhomogeneous diffusion simulation program developed and provided by Siggia et al. (2000).

Mouse lines. Transgenic mice expressing NF186-EGFP, NF186ecto-ICAM1cyto- (NF/ICAM)-EGFP, and ICAM1ectoNF186cyto-(ICAM/NF)-EGFP under the control of the Thy-1.2 promoter were generated as described previously (Y. Zhang et al., 2012). Caspr KOs have also been previously described (Feinberg et al., 2010) and were used to generate the requisite crosses. All experiments were performed in compliance with the relevant laws and institutional guidelines and were approved by the New York University School of Medicine Animal Care and Use Committee.

Experimental design and statistical analysis. To analyze the mechanisms by which NF186 accumulates at nodes, we compared the targeting of WT and mutant NF186 constructs expressed in cultured neurons or transgenically in WT versus Caspr mutant mice. In general, three or four pairs of WT and Caspr KO mice expressing different transgenes were analyzed. We characterized equivalent numbers of male and female mice; there was no gender difference in how WT and chimeric constructs were targeted. For P3 sciatic nerves, 250-350 ankyrin $\mathrm{G}^{+}$ nodes were analyzed for each condition and for adult sciatic nerves, 150-200 ankyrin $\mathrm{G}^{+}$nodes were analyzed for each condition. In the case of optic nerves, $500-800$ ankyrin $\mathrm{G}^{+}$or $\beta \mathrm{IV}$ spectrin $^{+}$nodes were analyzed for each condition. For nodes expressing constructs, the intensity was measured and statistical analysis of the intensity distribution was done with unpaired Student's $t$ test. Unless otherwise stated, all data are represented as mean $\pm \mathrm{SD}$ and were analyzed using Prism software. To analyze the barrier function of the PNJs, we performed FRAP along internode, paranode, and node with intact or compromised PNJs. The diffusion coefficient was determined using an inhomogeneous diffusion simulation program developed and provided by Siggia et al. (2000). We only retained results generated from experiments in which the experimental and simulation recovery curves matched. In general, four to six experimental results were averaged, and SEM was calculated (Table 1).

\section{Results}

\section{The paranodes regulate targeting of NF186 to mature PNS nodes in vitro}

As nodes mature in the PNS postnatally, NF186 targeting (i.e., its accumulation at nodes), switches from diffusion trapping mediated by its ectodomain to a transport-dependent mechanism that relies on its cytoplasmic segment (summarized in Fig. 1). Node maturation and this switch in targeting are correlated with the assembly of the flanking paranodes (Y. Zhang et al., 2012), suggesting they regulate this transition. To address this possibility directly, we compared targeting of NF186 constructs in WT versus Caspr KO neurons (Gollan et al., 2003), which have defective PNJs (Bhat et al., 2001), using myelinating cultures (Fig. 1Ac). We analyzed three distinct neurofascin constructs each tagged with a GFP reporter (Fig. 1B): WT NF186-EGFP, NF/ICAM- 
A

a diffusion trapping

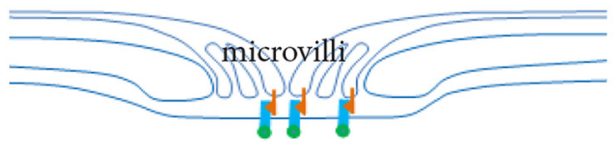

b transport-dependent

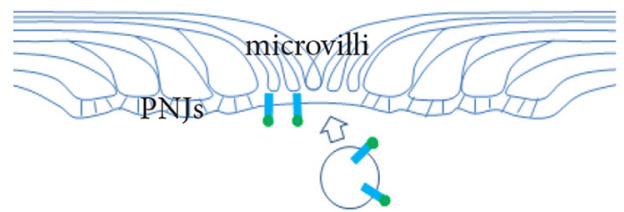

C

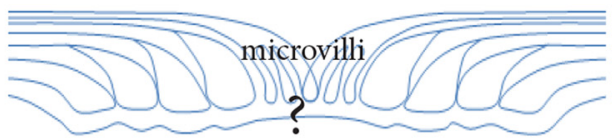

B

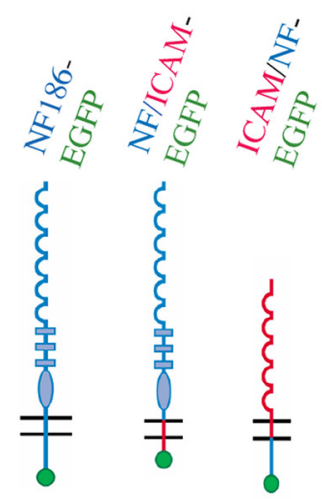

C a

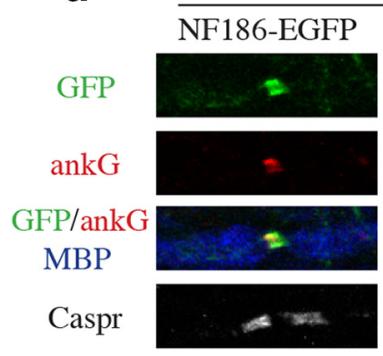

b

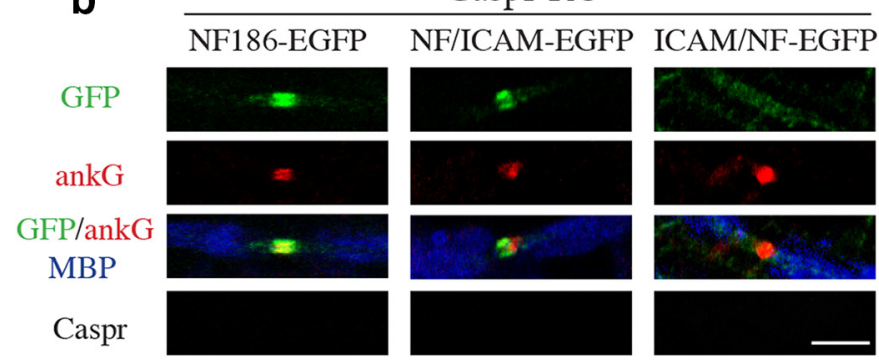

C

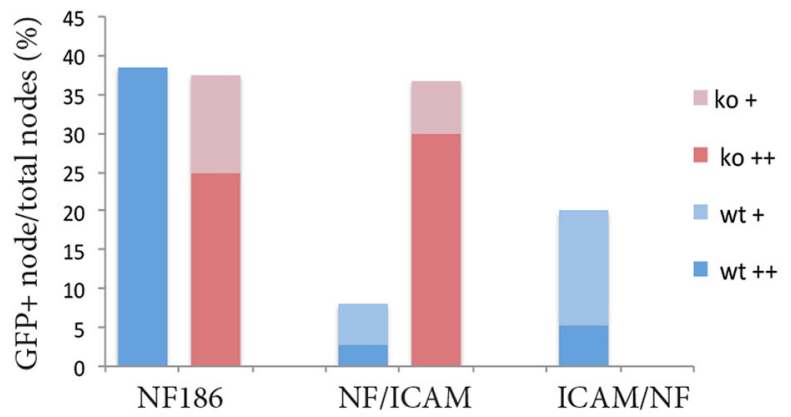

Figure 1. Targeting of NF186 constructs to mature nodes in vitro is regulated by the paranodes. $\boldsymbol{A}$, Schematic of NF186 targeting mechanisms. Initial targeting of NF186 to PNS nodes (Aa) relies on diffusion trapping mediated by interactions of its ectodomain with glial ligands (i.e., gliomedin), whereas targeting to mature nodes, flanked by PNJs (A $\boldsymbol{b}$ ), is mediated by its cytoplasmic domain and is transport-dependent (with NF186 present in carrier vesicles). This study examines targeting to the nodes driven by the NF186 ectodomain and the NF186 cytoplasmic domain in the absence of PNJS (AC). B , Schematic showing NF186 targeting constructs used in this study. These include WT NF186 and two chimeric constructs: the NF186 ectodomain fused to ICAM1 cytoplasmic domain (NF/ICAM) and the ICAM1 ectodomain fused to neurofascin cytoplasmic domain (ICAM/NF). All constructs have a GFP-tag at the C-terminus. Blue represents sequences from neurofascin. Red represents sequences from ICAM1. Green represents sequences from EGFP. C, Expression of WT neurofascin and chimeric constructs was induced in established, myelinated cocultures with (Ca) WT and (Cb) Caspr KO neurons. Cultures were stained for GFP (green), AnkG (red), MBP (blue), and Caspr (white). Scale bar, $5 \mu$ m. Quantification of a representative set of cultures is shown in $\mathbf{C c}+,++$ indicate relative brightness.

EGFP in which the neurofascin ectodomain is fused to the ICAM1 cytoplasmic domain, and ICAM/NF-EGFP in which the ICAM1 ectodomain is fused to the neurofascin cytoplasmic domain. ICAM1 is a lymphocyte cell adhesion molecule that exhibits a diffuse and nontargeted distribution when ectopically expressed in neurons (Dzhashiashvili et al., 2007). Thus, NF/ ICAM-EGFP specifically reports NF186 ectodomain-dependent targeting, whereas ICAM/NF-EGFP reports NF186 cytoplasmic domain-dependent targeting (Y. Zhang et al., 2012).

We compared targeting of these constructs in WT versus Caspr KO DRG neurons cocultured with Schwann cells under myelinating conditions. Neurons were infected with lentiviral constructs (pSLIK) to allow inducible expression of these constructs. Schwann cells were then added, and cultures were allowed to myelinate for 4-6 weeks before inducing expression of the constructs with doxycycline. As myelination is nearly complete at this time, nodes were largely mature (i.e., flanked on both sides by PNJs).

We found control NF186 was targeted equivalently to mature nodes of WT and Caspr KO neurons (Fig. $1 \mathrm{Ca}-\mathrm{Cc}$, NF186).
Consistent with our previous report (Y. Zhang et al., 2012), targeting of the ectodomain-dependent NF/ICAM construct to mature nodes with normal PNJs was limited. In contrast, targeting of this NF/ICAM construct to nodes with defective paranodes in the Caspr $\mathrm{KO}$ cocultures was robust (Fig. $1 \mathrm{Ca}-\mathrm{Cc}, \mathrm{NF} /$ ICAM), indicating the PNJs normally prevent ectodomain-dependent clustering of NF186. Strikingly, the opposite findings were observed for cytoplasmic domain-dependent targeting of the ICAM/NF construct. This construct was targeted to nodes with intact paranodes on both sides but clustering was essentially lost in the Caspr KO neurons, which lack effective PNJs (Fig. $1 \mathrm{Ca}-\mathrm{Cc}, \mathrm{ICAM} / \mathrm{NF}$ ). These results indicate that intact PNJs are required for the cytoplasmic domain-dependent nodal accumulation of NF186.

The paranodes regulate NF186 targeting to forming PNS nodes in vivo

To assess the role of the paranodes in vivo, we examined targeting of these NF186 constructs, expressed as transgenes in 
A a

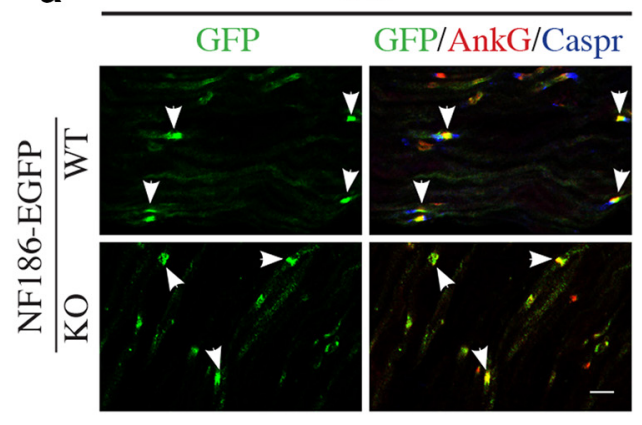

B a

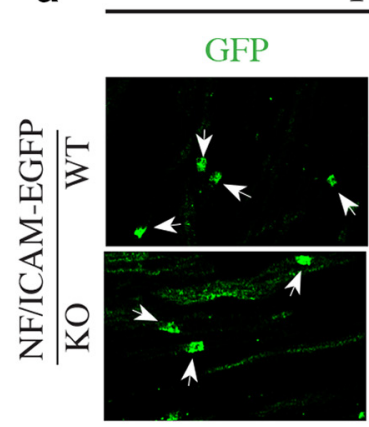

P3
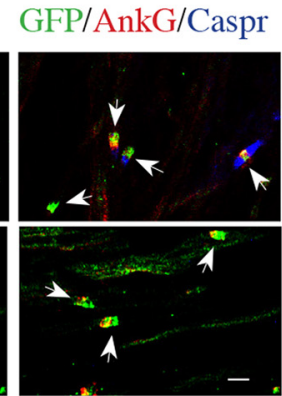

C a

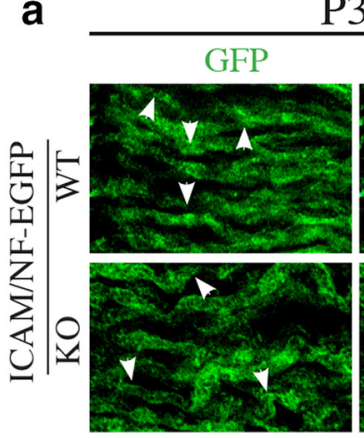

b

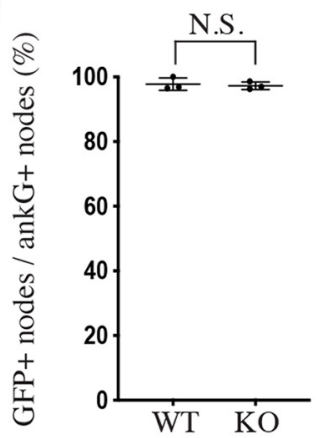

b

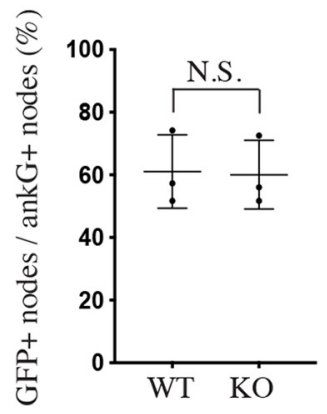

b GFP/AnkG/Caspr
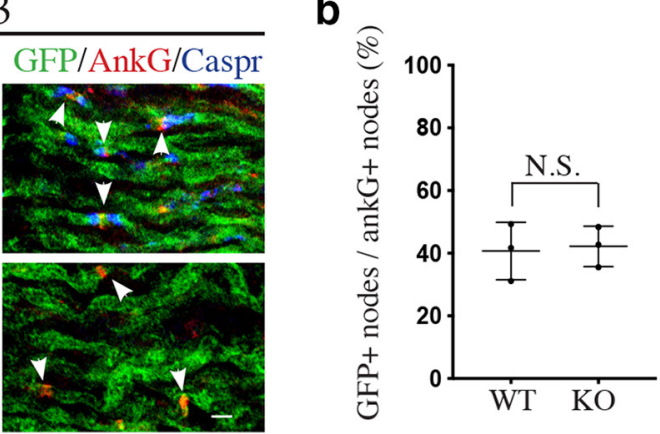

C

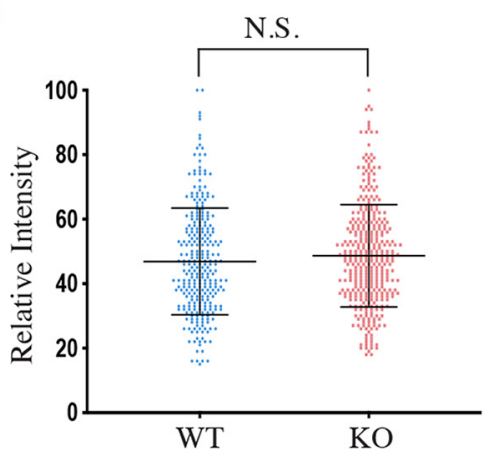

C

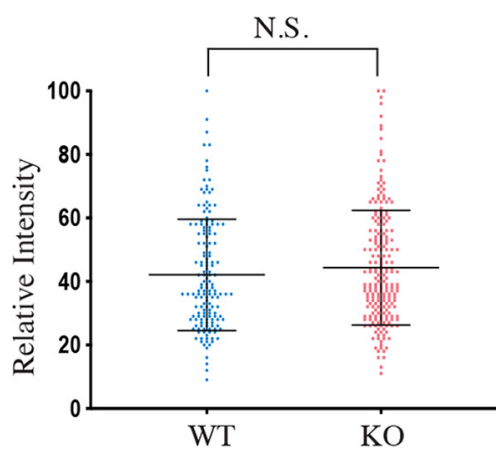

C

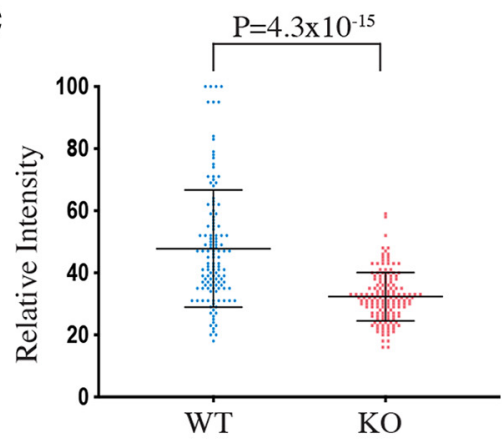

Figure 2. Targeting of neurofascin constructs to P3 nodes in WT and Caspr KO sciatic nerves. The targeting of neurofascin transgenic constructs to forming PNS nodes is shown for the following: (A) WT NF186, (B) NF/ICAM, and (C) ICAM/NF in WT and Caspr null backgrounds. For each case, (a) representative immunofluorescence images, $(\boldsymbol{b})$ quantification of GFP ${ }^{+}$nodes as a percentage of ankyrin $\mathrm{G}^{+}$nodes, and (c) the distribution of relative intensities of $\mathrm{GFP}^{+}$nodes are shown. $\boldsymbol{b}$, $\boldsymbol{c}$, Results show mean \pm SD averaged from three or four pairs of WT and Caspr KO nerves in each condition; $(\boldsymbol{b})$ each dot represents the result from 1 animal, and $(\boldsymbol{c})$ each dot represents the relative intensity at a single node. Paired $(\boldsymbol{b})$ and unpaired $(\boldsymbol{c}) t$ tests were used to assess significance. White arrowheads in the micrographs indicate location of nodes. Scale bar, $5 \mu \mathrm{m}$. N.S., not significant.

neurons under control of the Thy 1.2 promoter as previously described (Y. Zhang et al., 2012), in WT and Caspr null mice (Feinberg et al., 2010). We first examined targeting of these constructs to PNS nodes in P3 sciatic nerves (Fig. 2). WT NF186 was robustly detected at nearly all nodes in both WT and Caspr KO nerves with comparable intensity, although there was a trend to stronger signal levels in the Caspr nulls (Fig. $2 A a-A c$ ). The NF/ ICAM construct was also robustly nucleated at $\sim 60 \%$ of all $\mathrm{AnkG}^{+}$nodes in both WT and Caspr KO mice with similar intensities, although again there was a trend to stronger signal levels in the Caspr nulls (Fig. $2 B a-B c$ ). These results are consistent with our previous findings that initial PNS node formation relies on ectodomain-dependent targeting that occurs before formation of the PNJs (Y. Zhang et al., 2012).

In contrast, the ICAM/NF construct was only faintly enriched at PNS nodes, and only at $\sim 40 \%$ of them (Fig. $2 \mathrm{Cb}$ ). This construct was also diffusely expressed at low levels along the axon
(Fig. 2Ca) as we have reported previously reflecting impaired clearance of this construct (Y. Zhang et al., 2012). Further, of those nodes that were $\mathrm{GFP}^{+}$, the intensity of ICAM/NF was significantly lower in the Caspr nulls than in WT mice (Fig. 2Cc; $\left.p=4.3 \times 10^{-15}\right)$. These results suggest that the cytoplasmic domain-dependent accumulation of NF186 is limited in early development and is normally promoted by the PNJs.

\section{The PNJs regulate NF186 targeting to mature PNS nodes in} vivo

We next examined the effects of the PNJs on targeting of NF186 constructs in the adult PNS. WT NF186 accumulated efficiently and equivalently at mature nodes of both WT and Caspr KO sciatic nerves (Fig. $3 A a-A c$ ). In contrast, NF/ICAM poorly nucleated adult WT nodes, being detected at only $\sim 38 \%$ of $\mathrm{AnkG}^{+}$nodes and at those only faintly (Fig. $3 B a-B c$ ). Of note, 
NF/ICAM accumulation at adult nodes was substantially restored in the Caspr nulls, being detected at nearly $90 \%$ of nodes and at these sites quite robustly (Fig. $3 B a-B c$ ). This increase in $\mathrm{NF} / \mathrm{ICAM}$ signal intensity at Caspr $\mathrm{KO}$ nodes was significant (Fig. $3 B a-B c, p=0.0003$ ).

The opposite pattern of targeting was observed with the ICAM/NF transgenics. Although ICAM/NF did not accumulate effectively at early nodes (Fig. 2C), it was robustly detected at nearly all adult WT nodes (Fig. $3 \mathrm{Cb}$ ). In addition to its localization to nodes, ICAM/NF persists at low levels along the entire axon, reflecting impaired clearance of this construct (Y. Zhang et al., 2012). Further, its accumulation at nodes was substantially lost in the Caspr KOs (Fig. $3 C b$ ), with only $\sim 50 \%$ of nodes detectably positive and where its intensity was markedly reduced (Fig. 3Cc); its extranodal expression was similar in WT and Caspr KOs. This marked attenuation of ICAM/NF in the Caspr KOs nodes contrasts with the robust nucleation of all major PNS node components at these sites, for example, AnkG (Fig. $3 A-C$ ), $\beta \mathrm{IV}$ spectrin, and $\mathrm{NaV}$, as previously described (Rios et al., 2003).

To explore the possibility that differences in transgene expression might affect the observed results, we checked their expression levels in the PNS. All three transgenic constructs were similarly expressed in $\sim 30 \%-40 \%$ of the large DRG neurons (data not shown). Based on Western blotting to the EGFP tag (Fig. 3D), sciatic nerve levels were comparable for NF186-EGFP and NF/ICAM-EGFP levels, whereas ICAM/NF-EGFP levels were much higher, reflecting its diffuse localization along the axon due to impaired clearance (Dzhashiashvili et al., 2007). Further, endogenous NF186 levels on sciatic nerve blots (Fig. 3E, black arrow) were similar across the different lines and were not affected by the loss of Caspr or the expression of transgenes.

Together, these results indicate that the PNJs are required for the switch in targeting of NF186 to PNS nodes from an initial ectodomain/diffusion-dependent to a subsequent cytoplasmic domain-dependent mechanism.

\section{NF186 targeting to CNS nodes is mediated by its cytoplasmic domain and is paranode-dependent}

We next examined targeting of NF186 constructs to forming and mature CNS nodes. To assess early CNS nodes, we examined P14 optic nerves, representing the onset of rapid myelination (Rasband et al., 1999). These early nodes were typically flanked by $\mathrm{Caspr}^{+}$paranodes; in some cases, Caspr staining was present without accumulation of AnkG or NF186 at the nodes (data not shown), consistent with prior reports that the paranodes precede CNS node assembly (Rasband et al., 1999). We first compared targeting of NF constructs with these early CNS nodes versus forming PNS nodes (Fig. 4). As described above, constructs containing the NF186 ectodomain (i.e., WT NF186 and NF/ICAM) accumulate efficiently at P3 PNS nodes, whereas the ICAM/NF construct does not (Fig. 4A-C, top). In the CNS, targeting of WT NF186 to P14 optic nerve nodes was similarly robust (Fig. $4 A$, bottom). However in contrast to the PNS, accumulation of ICAM/NF was substantial, whereas that of NF/ICAM was negligible (Fig. $4 B, C$, bottom).

These results indicate targeting of NF186 to early CNS nodes is largely driven by its cytoplasmic domain, and suggest this targeting requires the paranodes. To assess the role of the PNJs directly, we quantified and compared the targeting of neurofascin constructs in WT and Caspr KOs to both forming and mature optic nerve nodes (Fig. 5A,B). At P14, WT NF186 nucleated at WT and Caspr KO nodes equally well with no significant differences (Fig. 5Aa). Less than $10 \%$ of P14 nodes were positive for NF/ICAM at either WT or Caspr KO nerves Fig. 5Ab). In striking contrast, ICAM/NF strongly accumulated at P14 WT but not Caspr KO nodes (Fig. 5Ac). Results were very similar in the adult. NF186-GFP accumulated robustly and equivalently (i.e., $>85 \%$ of total nodes) in both WT and Caspr KOs (Fig. $5 \mathrm{Ba}$ ), despite the marked elongation of CNS nodes characteristic of the Caspr KOs, as we previously reported (Rios et al., 2003). Likewise, there was minimal accumulation of NF/ ICAM in the adult optic nerve nodes $(<10 \%)$ in both WT and Caspr KOs (Fig. 5Bb). Finally, ICAM/NF accumulated effectively at adult WT optic nerve nodes $(\sim 60 \%)$ but minimally in the Caspr KOs (Fig. $5 \mathrm{Bc}$ ). It is unlikely that the lack of accumulation of NF/ICAM at nodes is due to lack of expression by neurons as comparable levels of the three different transgenes were detected in optic nerve protein lysates (Fig. 5C).

Thus, NF186 targets to both forming and mature CNS nodes mainly via its cytoplasmic domain, similar to the pattern observed in mature PNS nodes, and which is consistent with a transport-dependent mechanism. The targeting by the cytoplasmic domain of NF186 in both the PNS and CNS requires intact PNJs. In contrast to the PNS, the contribution of an ectodomaindependent, diffusion trapping mechanism appears limited both during development and in the adult CNS, even in the absence of the PNJs. However, as WT NF186 accumulation was normal in the Caspr KOs whereas ICAM/NF was disrupted, the NF ectodomain may cooperate with the NF cytoplasmic domain to promote recruitment and/or stability at CNS nodes.

\section{The PNJs function as a diffusion barrier}

A key question is how do the PNJs regulate targeting to PNS and CNS nodes. The enhanced accumulation of NF/ICAM at PNS nodes in the Caspr nulls is suggestive of loss of a paranodal barrier, thereby allowing this construct to diffuse from extranodal sites to the node. To test this possibility directly, we performed FRAP analysis with several GFP constructs. These were expressed in DRG neurons under myelinating conditions; in WT cultures, we also coexpressed hCaspr-mKate2 to demarcate the paranodes.

We first analyzed the diffusion of ICAM1-EGFP, which has a membrane topology similar to NF186 (i.e., it is a transmembrane protein with extracellular Ig-like domains). However, in contrast to NF186, ICAM1 lacks interacting ligands on myelinating Schwann cells or within the axon and is therefore diffusely expressed along axons before and after myelination (Fig. 6) (Dzhashiashvili et al., 2007; Y. Zhang et al., 2012). ICAM1 levels are partially reduced at WT paranodes (Fig. 6B), suggesting a local barrier and/or steric effects of other proteins enriched at this site of close axoglial apposition. In agreement, ICAM1 expression along the axon is uniform in the Caspr $\mathrm{KO}$ under myelinating conditions, with no reduction in the paranodal regions immediately adjacent to the nodes (Fig. 6C). FRAP analysis shows ICAM1 is freely diffusible in the internode of WT axons (Fig. 6A; Table 1). Bleaching of ICAM1-GFP at the node revealed limited recovery in WT neurons, whereas there was rapid recovery in the Caspr KOs (Fig. 6B,C; Table 1). These results corroborate that the paranodes represent a barrier to the diffusion of transmembrane proteins.

We next examined the subcellular location of this diffusion barrier. Recent studies indicate the axonal cytoskeleton at the paranodes has a key role in segregating nodal from juxtaparanodal proteins, suggestive of an intracellular barrier (Amor et al., 2017). To test for an intracellular barrier directly, we conducted 
A a

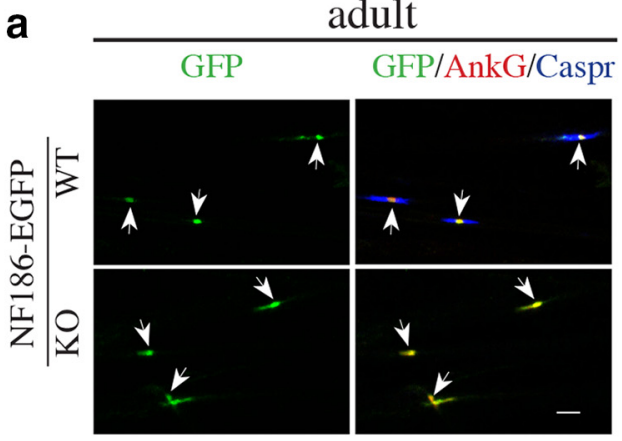

B a

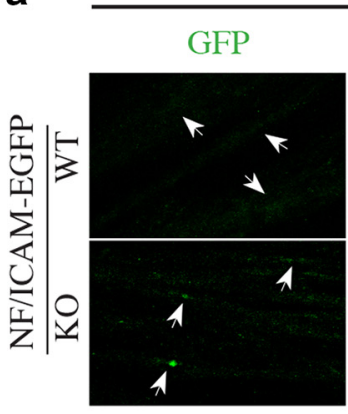

adult
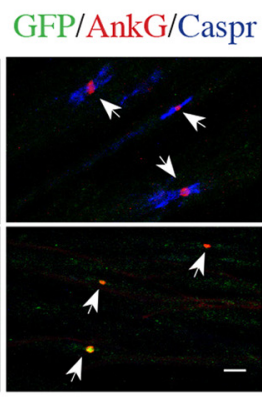

C a adult

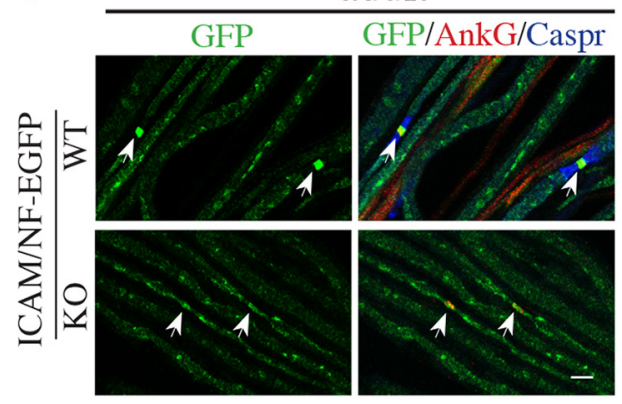

b

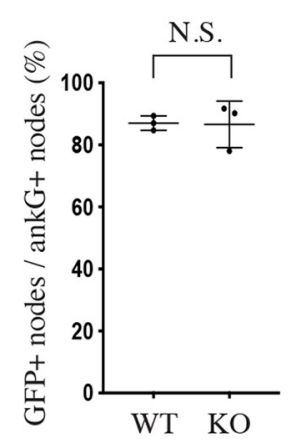

b

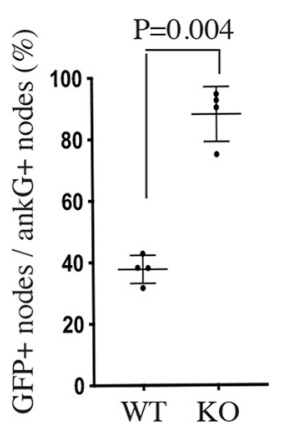

b

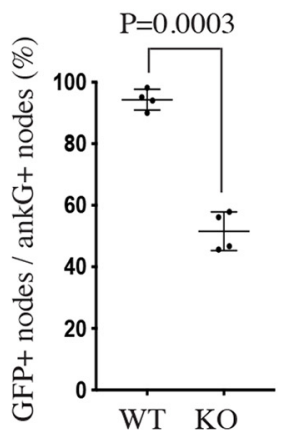

C

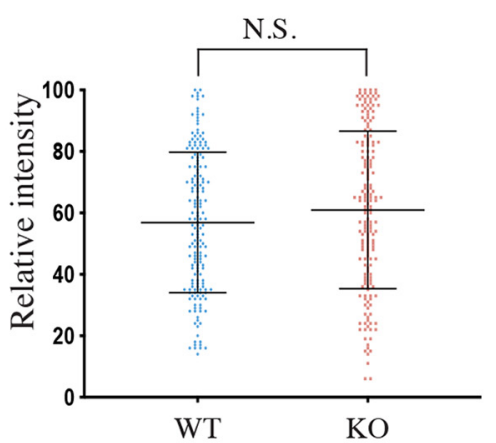

C

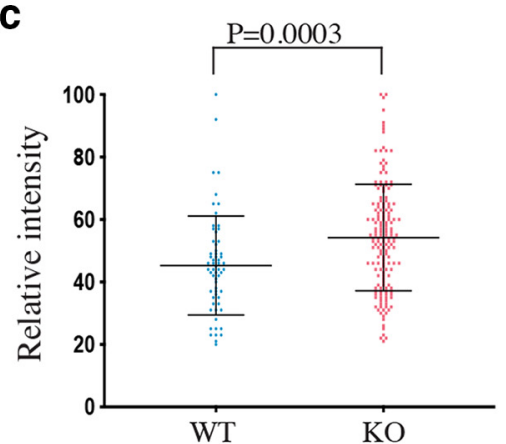

C

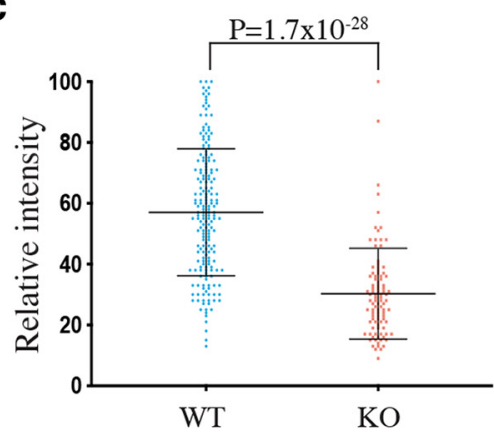

D

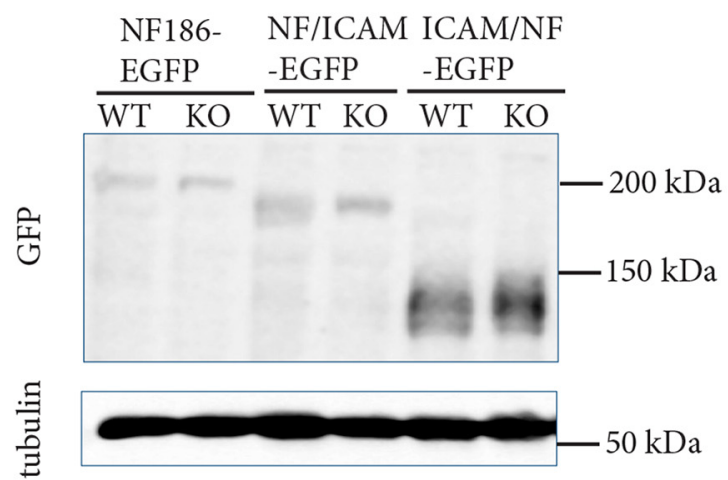

E

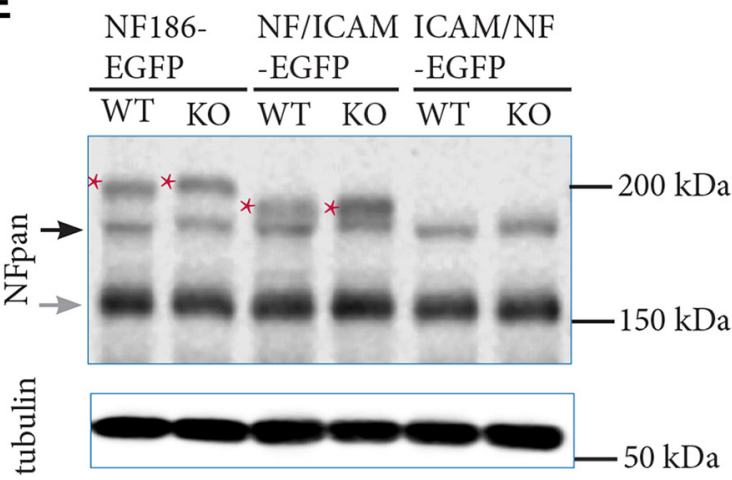

Figure 3. Targeting of neurofascin constructs to adult nodes in WT and Caspr KO sciatic nerves. Representative images show targeting of neurofascin transgenic constructs to adult PNS nodes for the following: (A) WT NF186, (B) NF/ICAM, and (C) ICAM/NF in WT and Caspr null backgrounds. In each case, (a) representative immunofluorescence images, (b) quantification of GFP ${ }^{+}$ nodes as a percentage of ankyrin $\mathrm{G}^{+}$nodes, and $(\boldsymbol{c})$ the distribution of relative intensities of $\mathrm{GFP}^{+}$nodes are shown. $\boldsymbol{b}, \boldsymbol{c}$, Results represent mean \pm SD averaged from three or four pairs of WT and Caspr KO nerves in each condition. $\boldsymbol{b}$, Each dot represents the result from 1 animal. $\boldsymbol{c}$, Each dot represents the relative intensity at a single node. Paired $(\boldsymbol{b})$ and unpaired $(\boldsymbol{c}) t$ tests were used to assess significance. White arrowheads in the micrographs indicate location of nodes. Scale bar, $5 \mu \mathrm{m}$. $\boldsymbol{D}, \boldsymbol{E}$, Western blots of the expression of transgenes $(\boldsymbol{D}, \boldsymbol{E})$ and endogenous neurofascin expression $(\boldsymbol{E})$ in P19 WT and Caspr K0 sciatic nerves. Tubulin served as a loading control $(\boldsymbol{D}, \boldsymbol{E})$. $\boldsymbol{D}$, Transgenic constructs were detected by probing with an anti-GFP antibody. $\boldsymbol{E}$, The blot was probed with an antibody (NFpan) that recognizes the ectodomain of neurofascin, including endogenous NF186 (black arrow), NF155 (gray arrow), and EGFP-tagged transgenes (red asterisks). ICAM/NF-EGFP is not detected by this NF-ectodomain antibody. As NF/ICAM-EGFP has a similar molecular weight to endogenous NF186, their corresponding bands migrate similarly. Endogenous NF186 levels are similar in the sciatic nerves of transgenic WT and Caspr null mice. N.S., not significant. 
A
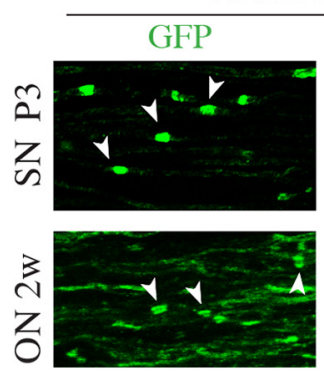

B

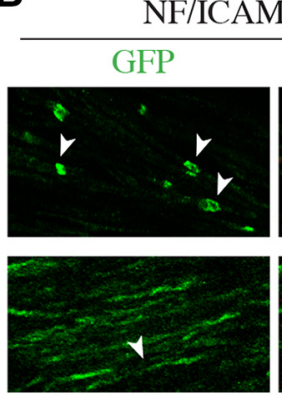

C

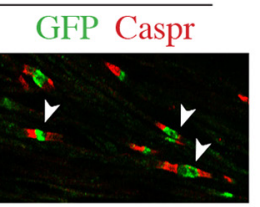

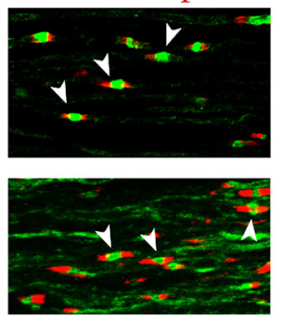

GFP Caspr

Figure 4. NF186 is targeted to forming PNS versus CNS nodes by different mechanisms. Representative images of the expression of different transgenic neurofascin constructs ( $\boldsymbol{A}-\boldsymbol{C}$ ) in early PNS (P3 sciatic) and CNS (P14 optic) nerves are shown in the top and bottom rows, respectively. Nerves are stained for GFP (green) and Caspr (red). White arrowheads indicate the positions of nodes. Of note, NF/ICAM-EGFP nucleates nodes of P3 sciatic nerves but not P14 optic nerves, whereas ICAM/NF-EGFP is minimally enriched at P3 sciatic nerve nodes but strongly accumulates at P14 optic nerve nodes. Scale bar, $5 \mu \mathrm{m}$.

a FRAP analysis of myristoylated GFP, a construct that binds to the inner leaflet of the plasma membrane via its acyl tag (Resh, 1999). MyrGFP was ubiquitously distributed along the membrane of myelinated axons (Fig. 6D). FRAP analysis indicates this construct is freely diffusible along the internodal axolemma with a diffusion coefficient of $0.38 \pm 0.02 \mu \mathrm{m}^{2} / \mathrm{s}$ (Fig. $6 D$; Table 1 ).

In contrast, when we bleached myrGFP at WT nodes, there was little if any recovery of fluorescence (Fig. 6E; Table 1), strongly suggesting there is a diffusion barrier at the level of the intracellular paranodal membrane. To test this directly, we conducted FRAP of myrGFP at the nodes of Caspr KOs, which were identified by expression of NF186-mCherry and phase-contrast images showing bilateral myelin sheaths (Fig. $6 F$ ). MyrGFP fluorescence recovered with a diffusion coefficient of $0.38 \pm 0.02$ $\mu^{2} / \mathrm{s}$, comparable with that along the internode, confirming the paranodes normally present a diffusion barrier that is lost in the Caspr KOs. FRAP analysis of myrGFP at a node flanked by only one fully formed paranode exhibited an intermediate rate of recovery (Fig. 6G), with a diffusion coefficient in between that of WT nodes and internodes (i.e., $0.20 \pm 0.04 \mu \mathrm{m}^{2} / \mathrm{s}$ ) (Table 1). These results suggest fluorescence recovery results from diffusion from the side of the node without PNJs. Finally, we photobleached myrGFP within the WT paranode itself, which resulted in essentially no fluorescence recovery over several minutes (Fig. 7). Together, these results confirm there is a barrier to the diffusion of membrane components across and within WT paranodes.

Changes in the cytoskeleton at the paranodes of Caspr nulls A likely component of the paranodal diffusion barrier is the submembranous cytoskeleton (Horresh et al., 2010; C. Zhang et al., 2013; Amor et al., 2017). In particular, Caspr is bound to $4.1 \mathrm{~B}$ (Gollan et al., 2002; Denisenko-Nehrbass et al., 2003), which in turn interacts with $\alpha \mathrm{II} / \beta$ II spectrin (Hoover and Bryant, 2000) and thereby the associated actin cytoskeleton. We characterized whether the organization of these cytoskeletal components is affected in the Caspr KOs comparing their expression in myelinated axons of WT versus Caspr KOs. In WT cultures, as previously reported (Horresh et al., 2010; Buttermore et al., 2011; Einheber et al., 2013), 4.1B is located in the paranode, where it is variably enriched, and along the internode; it is excluded from WT nodes (Fig. 8A). In Caspr KO cultures, 4.1B immunoreactivity level was specifically attenuated in the paranodal region (Fig. $8 A$ ). Similar findings were observed in sciatic nerves from WT and Caspr $\mathrm{KO}$ mice (Fig. 8B). In WT nerves, 4.1B exhibits a sharp border between the paranodes and node and is enriched at some paranodes. In Caspr KO nerves, 4.1B staining was generally reduced at the paranodes and the boundary between the node and paranode was indistinct compared with WT nerves; $4.1 \mathrm{~B}$ expression along the internode was unaffected.

As $4.1 \mathrm{~B}$ interacts with both Caspr and $\beta$ II spectrin, Caspr is a candidate to stabilize $4.1 \mathrm{~B}$ expression at paranodes. To test this, we treated live cultures with $0.5 \%$ Triton X-100 to remove nonstabilized components of the cytoskeleton, then fixed and immunostained cultures. Extracting WT cultures did not reduce 4.1B in the paranodes, where it colocalized with Caspr, but partially removed it from the internodes (Fig. 8C, WT). In contrast, $4.1 \mathrm{~B}$ in the paranodes was largely gone after Triton X-100 extraction of Caspr $\mathrm{KO}$ cultures (Fig. 8C, KO). Similar findings were observed in sciatic nerves after Triton X-100 extraction (Fig. $8 D$ ). These results strongly suggest that Caspr normally stabilizes $4.1 \mathrm{~B}$ at the paranodes and accordingly that the persistent but reduced levels of $4.1 \mathrm{~B}$ in the Caspr KOs are not appropriately tethered.

We next examined $\beta$ II spectrin expression in sciatic nerves. As shown in Fig. $8 E, \beta$ II spectrin is present at the paranodes and internodes and excluded from the nodes in WT nerves similar to that of 4.1B. There is also substantial expression in Schwann cells (i.e., the outer cytoplasmic collar) in agreement with a previous report (Einheber et al., 2013). The distribution of $\beta$ II spectrin was not altered in Caspr KO nerves (Fig. $8 E$ ), but the overall intensity was modestly elevated. Western blotting revealed enhanced expression of a lower molecular weight $\beta$ II spectrin band in lysates from the PNS of Caspr KOs (Fig. 8G) that may correspond to a proteolytic fragment and account for the slight increase in staining. Triton X-100 extraction of WT and Caspr KO mice sciatic nerves did not alter the distribution of $\beta$ II spectrin and normalized the levels detected by staining of WT versus KO nerves (Fig. 8F). Together, loss of Caspr dramatically destabilizes accumulation of $4.1 \mathrm{~B}$, but not $\beta$ II spectrin, in the paranodes. These results suggest the paranodal barrier requires proper linkage between the Caspr/contactin membrane adhesion complex and the underlying $4.1 \mathrm{~B}$ based cytoskeleton.

\section{Discussion}

The paranodes regulate a switch in targeting of NF186 to PNS nodes

The assembly of nodes of Ranvier is essential for the acquisition of saltatory conduction. Two distinct but complementary neuron-glia interactions promote node assembly and its stability: (1) interactions of NF186 at the node with glial receptors (PNS) or 
A

P14

a

NF186-EGFP

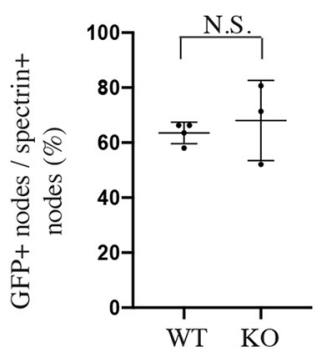

b

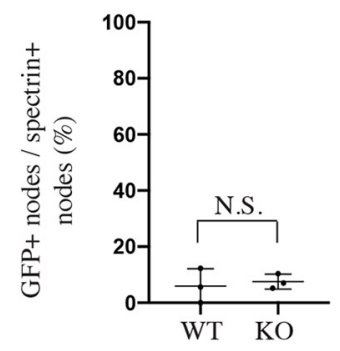

C

ICAM/NF-EGFP

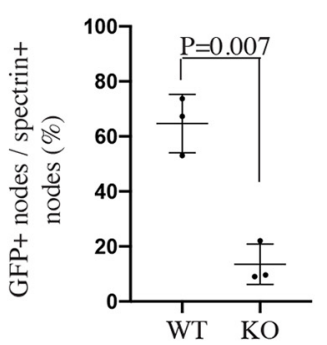

B

\section{a}

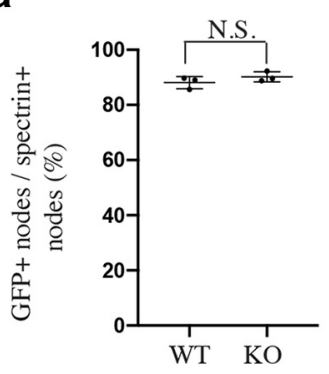

b

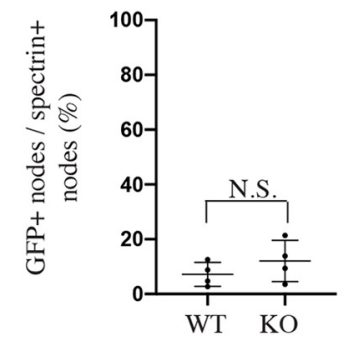

C

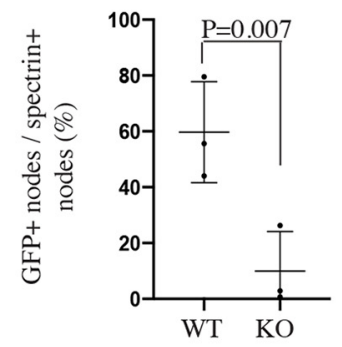

C

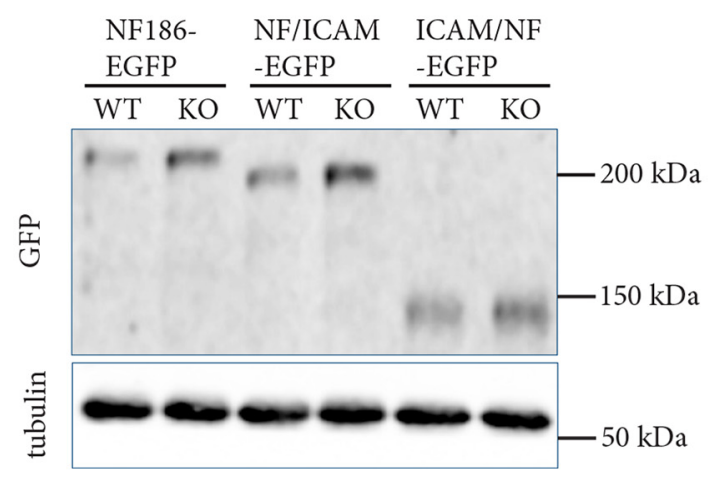

Figure 5. Quantification of the targeting of neurofascin constructs to nodes of WT and Caspr KO optic nerves. The percentage of $\mathrm{GFP}^{+}$nodes/total $\beta$ IV spectrin ${ }^{+}$nodes for $(\boldsymbol{A})$ P14 and (B) adult optic nerves are shown for (a) WT NF186, (b) NF/ICAM, and (c) ICAM/NF transgenic constructs. Results represent mean \pm SD averaged from three or four pairs of WT and Caspr KO nerves in each condition. Each dot represents the result from 1 animal. The paired $t$ test was used to assess significance. WT NF186 (a) accumulated at WT and Caspr K0 nodes equally well in the 2 week and adult optic nerves, whereas NF/ICAM (b) minimally accumulated at nodes of WT and Caspr KO nerves in either age group. ICAM/NF (c) accumulated at WT nodes efficiently in both age groups but not in Caspr KO nodes $(p=0.007)$. $C$, Expression of the transgenic contructs in lysates prepared from P19 optic nerves. The blot was stained with an anti-GFP antibody to detect transgene expression; tubulin served as a loading control. N.S., not significant.

with glial extracellular matrix components (CNS), which serve to recruit/stabilize the complex; and (2) the paranodal barrier that delineates the node and prevent intrusion of juxtaparanodal components. Together, these drive the specialized ankyrin G/ $\beta$ IV spectrin cytoskeleton at the node (Rasband and Peles, 2015; Liu et al., 2020) that tethers and stabilizes the multimeric, nodal complex (channels, adhesion molecules).

The paranodes not only provide a barrier that surrounds the node; they also play important roles in its assembly. Thus, in the absence of NF186, the paranodes are able to drive PNS node assembly, albeit at reduced intensity (Amor et al., 2017). However the paranodes are not essential for node assembly as PNS and CNS nodes still assemble in their absence (Dupree et al., 1999; Bhat et al., 2001; Boyle et al., 2001; Zonta et al., 2008). The paranodes also sustain the integrity of the nodes, which disperse over time when the paranodes are disrupted, even after node assembly is complete (Rios et al., 2003; Pillai et al., 2009; Taylor et al., 2017). Thus, the paranodes share key roles in node assembly and maintenance.

A major question is how the paranodes contribute to node assembly. Here, we demonstrate that the paranodes regulate the mechanisms by which NF186 accumulates at nodes. In particular, our results support the notion that the paranodes are required for the switch in NF186 targeting to PNS nodes from diffusion-trapping (mediated by its ectodomain) to a transportdependent mechanism of accumulation (mediated by its cytoplasmic domain). A paranode-mediated switch was originally suggested by the fact that NF186 is targeted to forming nodes via its ectodomain (Dzhashiashvili et al., 2007; Y. Zhang et al., 2012), whereas it is later targeted to older, mature nodes via its cytoplasmic domain (Y. Zhang et al., 2012). We have confirmed this role using Caspr KO mice, which lack transverse bands and the tightly apposed glial loops characteristic of normal paranodes (Bhat et al., 2001). In Caspr KO mice, NF186 accumulation at mature PNS nodes continues to be driven by its ectodomain and fails to switch to cytoplasmic domain-dependent recruitment (Figs. 1C, 3). The lack of targeting by the NF186 cytoplasmic domain in the Caspr nulls is particularly striking as AnkG, to which this segment binds and is stabilized by, accumulates normally at nodes (Figs. 3, 8).

\section{NF186 accumulates at PNS and CNS node distinctly}

Our studies are also consistent with earlier reports that suggest important differences in PNS and CNS node assembly and the role of the paranodes. In the PNS, NF186 is recruited to forming nodes by interactions with gliomedin presented by the Schwann cell microvilli (Eshed et al., 2005), where it pioneers assembly of the nodal complex (Lambert et al., 1997; Sherman et al., 2005; Dzhashiashvili et al., 2007). The paranodes then form with a slight delay after initial PNS node assembly (Melendez-Vasquez et al., 2001). This sequence is essentially reversed in the CNS, where the paranodes form first followed by accumulation of the nodal cytoskeleton and then NF186 (Rasband et al., 1999; Jenkins and Bennett, 2002). In the CNS, NF186 interacts with and may recruit ECM components to nodes, which only accumulate after nodes have already formed; these ECM components are dispensable for node assembly and NF186 accumulation (Oohashi et al., 2002; Bekku et al., 2009; Susuki et al., 2013). Thus, whereas PNS node assembly is normally initiated by Schwann cell-dependent recruitment of NF186 (outside-in), CNS node assembly appears to assemble by organizing the cytoskeleton first, then recruiting NF186 and ECM components (inside-out) (Fig. 9). 
A WT_internode

a ICAM1-EGFP + hCaspr-mKate2

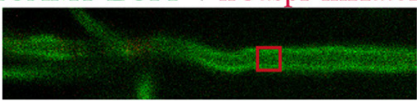

phase

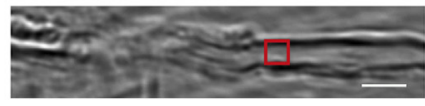

b
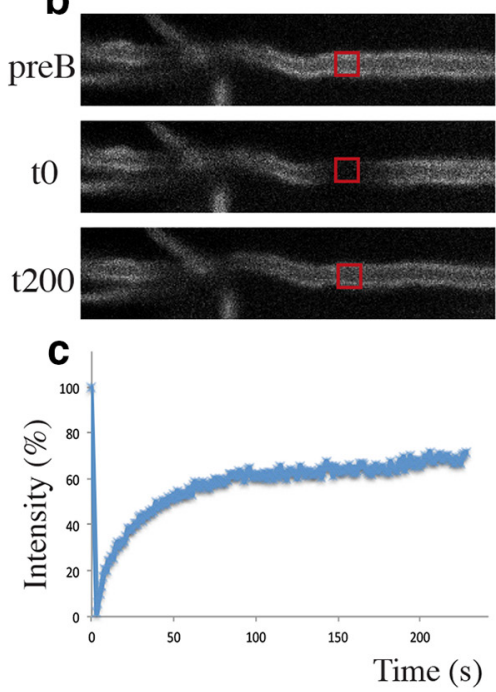

B WT_node

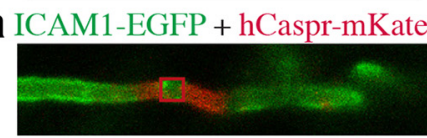

phase

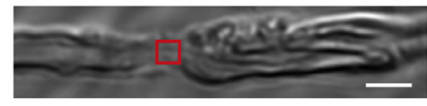

b
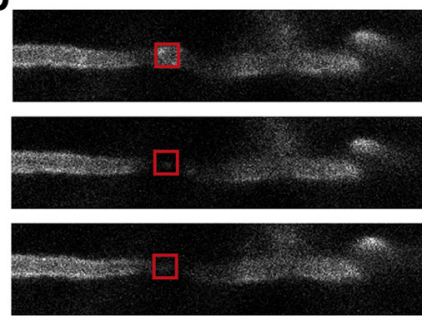

C

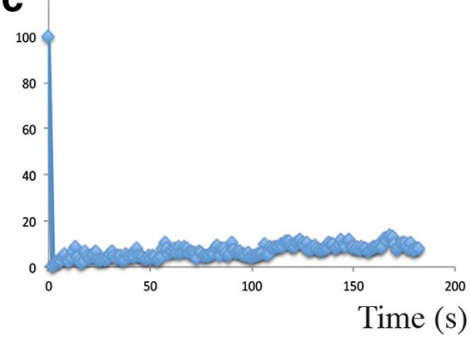

C Caspr KO_node

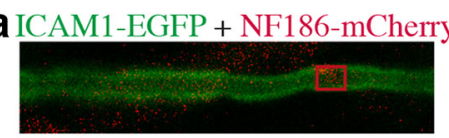

phase

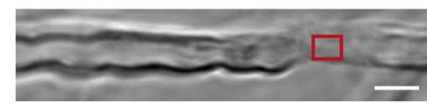

b

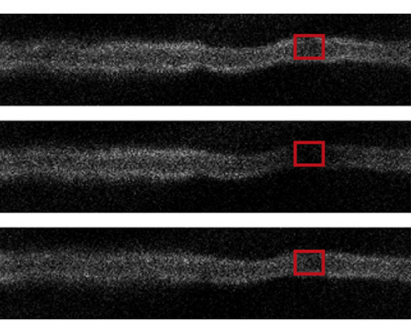

C

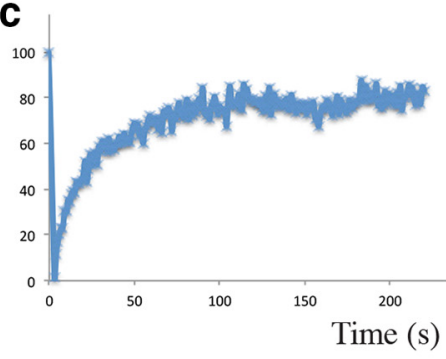

D WT internode E

a MyrEGFP + hCaspr-mKate2

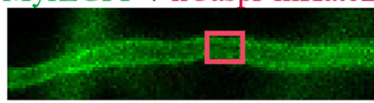

phase

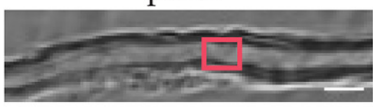

b

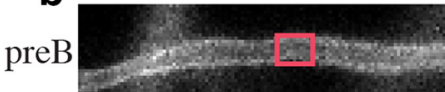

t0
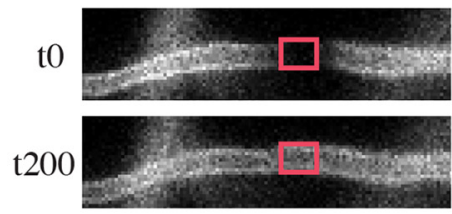

C

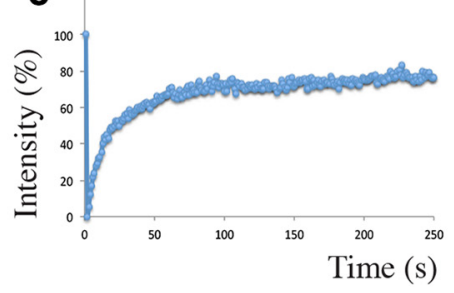

E WT_node

a MyrEGFP + hCaspr-mKate2

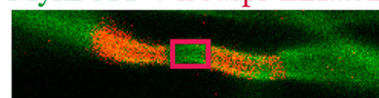

phase

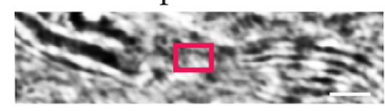

b
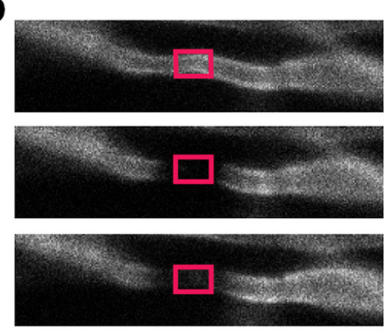

C

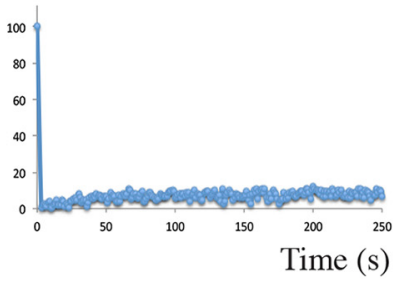

F Caspr KO_node

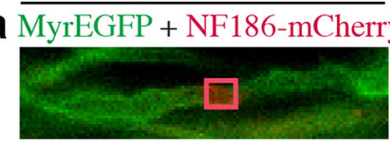

phase

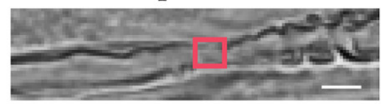

b
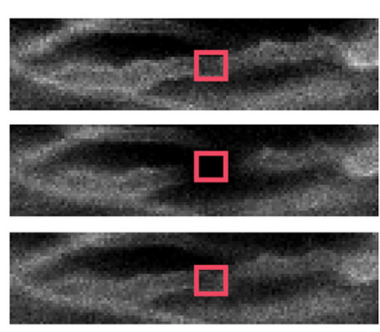

C

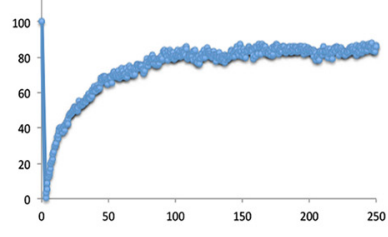

Time (s)
G WT heminode

a MyrEGFP + hCaspr-mKate2

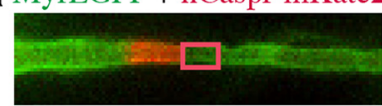

phase

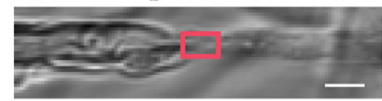

b
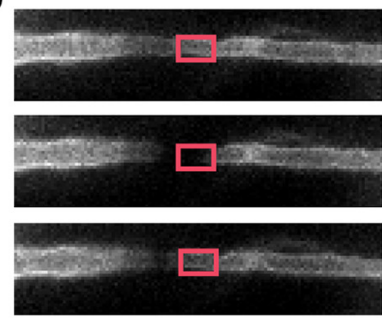

C

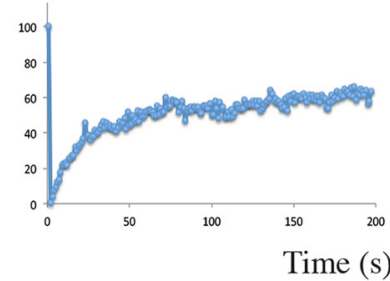

Figure 6. FRAP analysis demonstrates a paranodal diffusion barrier. $\boldsymbol{A}-\boldsymbol{C}$, FRAP analysis of ICAM1-EGFP and (D-G) FRAP analysis of MyrGFP in WT and Caspr KO myelinating cocultures. The paranodes in WT cocultures were demarcated by hCaspr-mKate2 (red); nodes in the K0 cocultures were demarcated by NF186-mCherry (red). Measurements of membrane diffusion for ICAM1GFP were taken from the following: $(\boldsymbol{A})$ the internode of WT axons, $(\boldsymbol{B})$ WT nodes, and (C) KO nodes. Diffusion measurements on MyrGFP were taken from the following: (D) the internode of WT axons, $(\boldsymbol{E})$ WT nodes, $(\boldsymbol{F})$ KO nodes, and $(\boldsymbol{G})$ WT heminode. $\boldsymbol{a}$, Red rectangles represent the positions of bleached areas; phase-contrast images represent myelin and positions of the nodes. $\boldsymbol{b}$, Sample images showing prebleach, immediately after bleach (t0), and $200 \mathrm{~s}$ after (t200) bleach. Scale bar, $5 \mu$ m. $\boldsymbol{c}$, Representative recovery curves for each condition are shown under the corresponding images. $y$ axis indicates the intensity as a percentage of the prebleached image. $x$ axis indicates time elapsed in seconds. 
Table 1. Summary of the FRAP analysis ${ }^{a}$

\begin{tabular}{llccl}
\hline & Bleach region & $D\left(\mu \mathrm{m}^{2} / \mathrm{s}\right)$ & SEM & $n$ \\
\hline ICAM1 EGFP & & & & \\
WT & Internode & 0.24 & 0.04 & 5 \\
WT & Node & $<0.01$ & - & 5 \\
K0 & Node & 0.23 & 0.02 & 6 \\
MyrEGFP & & & & \\
WT & Internode & 0.38 & 0.02 & 4 \\
WT & Node & $<0.01$ & - & 6 \\
WT & Heminode & 0.2 & 0.04 & 5 \\
KO & Node & 0.38 & 0.02 & 4 \\
\hline
\end{tabular}

${ }^{a}$ FRAP of ICAM1-EGFP and MyrEGP following photobleaching of different domains of myelinated axons in WT and Caspr KO neurons was used to calculate diffusion coefficients (D). Results are averaged from several experiments. These findings indicate that the paranodes are a barrier for the diffusion of a transmembrane (ICAM1) and a peripheral membrane (MyrEGFP) protein into nodes of Ranvier.

A
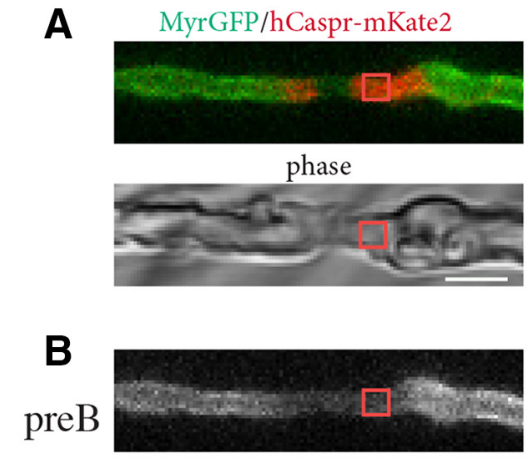

t0
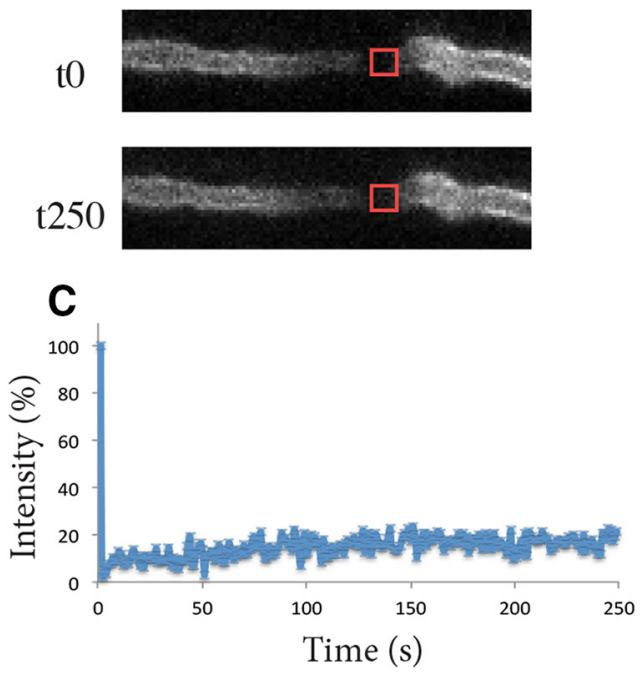

Figure 7. Membrane diffusion is limited within the paranodal region. FRAP analysis of MyrGFP within the paranode of a WT cultures. A, Representative images showing coexpression of MyrGFP (green) with hCaspr-mKate2 (red) within the paranodes. Red rectangles represent the position of the bleached area. The corresponding phase-contrast image is shown below. B, Levels prebleach, immediately after bleach (t0), and $200 \mathrm{~s}$ after bleach (t200). Scale bar, $5 \mu \mathrm{m}$. C, Representative recovery curve, with the $y$ axis showing the intensity as a percentage of the prebleached image and the $x$ axis as time elapsed in seconds. There is essentially no fluorescence recovery after photobleaching of MyrGFP at this paranode.

These differences in the sequence of domain assembly are reflected in distinct modes of NF186 targeting. Thus, NF186 is initially targeted to PNS nodes by ectodomain-mediated (Fig. 4, top) diffusion trapping, whereas in the CNS it is targeted via its cytoplasmic domain to both forming (Fig. 4, bottom) and mature nodes (Fig. $5 \mathrm{Bc}$ ). Even in the Caspr KOs, NF186 fails to be recruited efficiently to CNS nodes via its ectodomain (Fig. $5 B b$ ).
These latter results suggest interactions with ECM components at CNS nodes are not robust enough to cluster NF186. Like PNS nodes, targeting of the NF186 cytoplasmic domain (e.g., ICAM/ $\mathrm{NF}$ ) to CNS nodes requires intact PNJs (Fig. 5BAc,Bc), further highlighting a critical role of PNJs on node assembly. Unexpectedly, full-length NF186 is targeted equally well to WT and Caspr KO CNS nodes (Fig. 5Aa,Ba). This latter result suggests the extracellular and intracellular domains of NF186 may cooperate to promote targeting in the Caspr null, even if the ectodomain by itself is not sufficient.

\section{The paranodes provide a membrane diffusion barrier for axonal components}

The PNJs have long been considered barriers to the diffusion of proteins within the membrane. Freeze fracture studies (Rosenbluth, 1976; Bhat et al., 2001) and immunofluorescence staining of nodal sodium and juxtaparanodal potassium channels (Dupree et al., 1999) show a distinct boundary for membrane proteins on either side of the paranodes. This barrier function depends on the cis complex of Caspr and contactin on the axon (Bhat et al., 2001; Boyle et al., 2001; Pillai et al., 2009) or its link to the cytoskeleton (C. Zhang et al., 2013); loss of either results in Kv1.1 and 1.2 relocating from the juxtaparanodes to the paranodes.

Here we show, for the first time, that the paranodes provide a barrier to the diffusion of axonal membrane proteins. Thus, there is essentially no recovery at nodes following photobleaching of a transmembrane protein (ICAM1-GFP) or an acylated reporter that binds to the intracellular leaflet (MyrGFP) in the presence of the PNJs; in contrast, there is rapid recovery in the Caspr KOs (Fig. $6 C, F$ ). These results indicate the paranodes, by providing a barrier, preclude diffusion trapping of NF186 and likely other nodal components, as suggested previously (Y. Zhang et al., 2012). Diffusion trapping of NF186 at PNS nodes is therefore possible only before formation of the paranodes (Fig. $2 B$ ) or if the paranodes are defective (Figs. $1 C, 3 B$ ). The lack of a diffusion barrier likely accounts for accumulation of NF/ICAM in the PNS at forming nodes and at adult nodes in the Caspr KOs, respectively.

An important related question is the nature of the diffusion barrier itself. Diffusion of membrane proteins along lipid bilayers can be constrained by multiple factors, including the size of the diffusing species, molecular crowding (Frick et al., 2007; Ramadurai et al., 2009), interactions within membrane microdomains and the cytoskeleton (Lenne et al., 2006; Trimble and Grinstein, 2015). The PNJs may limit diffusion by both extracellular and intracellular mechanisms. In the former case, this may include steric effects imposed by the tight apposition of axonal and glial membranes at the paranodes (i.e., 3-5 nm). This spacing that may exclude axonal membrane proteins with large extracellular domains (e.g., NF186), although not peripheral membrane proteins or transmembrane proteins with small extracellular domains. As the close apposition between the paranodal loops and axolemma is lost in paranodal mutants (Bhat et al., 2001; Boyle et al., 2001; Pillai et al., 2009), any diffusion block resulting from this apposition would also be lost. Similarly, the adhesion complex at the paranodes may sterically hinder ("crowd") other proteins from entering this membrane domain and limit their lateral movement in WT but not Caspr KOs. In potential agreement, ICAM1-EGFP expression was notably reduced at WT but not Caspr KO paranodes (Fig. 6Bb, $\mathrm{Cb}$ ).

Intracellularly, the linkage of the paranodal complex to the cytoskeleton is also likely to contribute to the diffusion barrier. 
Electron tomography had previously identified short filamentous linkers/ cross-bridges that connect the paranodal membrane to the cytoskeleton (Perkins et al., 2008; Nans et al., 2011). These likely correspond to interactions of the Caspr/contactin complex with the underlying $\alpha \mathrm{II} /$ $\beta$ II spectrin and actin cytoskeleton, mediated by the adaptor protein 4.1B, which binds to the C-terminus of Caspr and to spectrin. In the Caspr nulls, 4.1B is partially reduced at the paranodes and is completely extracted by Triton X-100, whereas $\beta$ II spectrin persists (Fig. 8), highlighting disruption of $4.1 \mathrm{~B}$ interactions at this site. In addition to these interactions, spectrins are known to bind directly with phospholipids in the membrane (Boguslawska et al., 2014), which may further contribute to axolemma/cytoskeletal interactions.

Several lines of evidence implicate these axolemma/cytoskeletal interactions in barrier formation. In mice that express mutants of Caspr that lack the $4.1 \mathrm{~B}$ binding domain, paranodal adhesion is normal, but Kv1.2 channels mislocalize to the paranodes (Horresh et al., 2010) similar to that of Caspr nulls (Bhat et al., 2001). Likewise, $\beta$ II spectrin null mice have normal extracellular paranodal adhesion and transverse bands; nevertheless, Kv1.2 expression gradually intrudes into the paranode and nodes (C. Zhang et al., 2013). These results are consistent with the structure of Kv1.2, which has a small extracellular but a bulky cytoplasmic segment (Chen et al., 2010). In agreement with a submembranous barrier, we show here that the paranodes limit diffusion of the inner membrane leaflet tethered GFP (MyrGFP) (Fig. 6E), which is only $4.2 \mathrm{~nm}$ long and $2.4 \mathrm{~nm}$ in diameter (Ormö et al., 1996). While the diffusion of MyrGFP across (Fig. 6E; Table 1) and within (Fig. 7) mature PNJs is limited, it freely diffuses along the internode (Fig. 6D) and across the defective paranodes of the Caspr nulls (Fig. 6F). These results indicate the paranodes present a submembranous barrier to even small molecules. As $\beta$ II spectrin is also required for the ability of the paranodes to drive node assembly (Amor et al., 2017), these results suggest this submembranous barrier also promotes node assembly.

Together, these results suggest the diffusion barrier at the paranode requires interactions of the Caspr/contactin complex with the $\beta$ II spectrin-based cytoskeleton that is $4.1 \mathrm{~B}$-dependent. High-resolution imaging indicates the axonal cytoskeleton has a striking microarchitecture of periodic actin rings that are bridged and spaced by spectrin tetramers (Xu et al., 2013; Leterrier, 2016; Huang et al., 2017). These rings are in register with Caspr/ Contactin in the paranodes and their apposed glia ligands (D'Este et al., 2017), indicating a molecular complex and likely barrier function that extends extracellularly from the glial loops to the intracellular axonal cytoskeleton. In agreement, loss of Caspr destabilizes the 4.1B-based paranodal cytoskeleton in the absence of these interactions (Fig. 8). Of note, the Cadm proteins also bind to 4.1B (Yageta et al., 2002) along the internode (Maurel et al., 2007; Spiegel et al., 2007) and are therefore likely to be linked to $\beta$ II spectrin and the actin rings. Nevertheless, both ICAM and MyrGFP are freely diffusible within the internode. These latter results suggest that additional, paranode-specific components (e.g., AnkB and/or other components) are required to establish the paranodal barrier.

\section{Mechanisms by which the PNJs regulate NF186 targeting to nodes}

A major question raised by this study is how the paranodes regulate targeting of NF186, and by inference other components of the AnkG/NaV complex, to nodes. We had previously demonstrated that existing NF186 on the axon membrane can diffuse to forming (hemi)nodes (Y. Zhang et al., 2012) and now show this route is abrogated in mature nodes by the barrier function of the paranodes. We also previously showed, and provide further 
A a

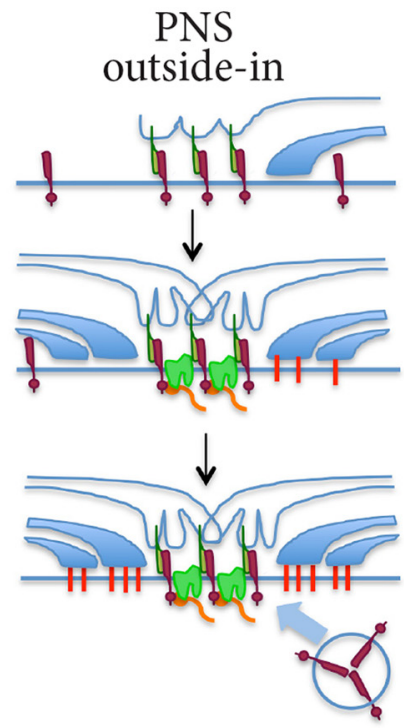

B a

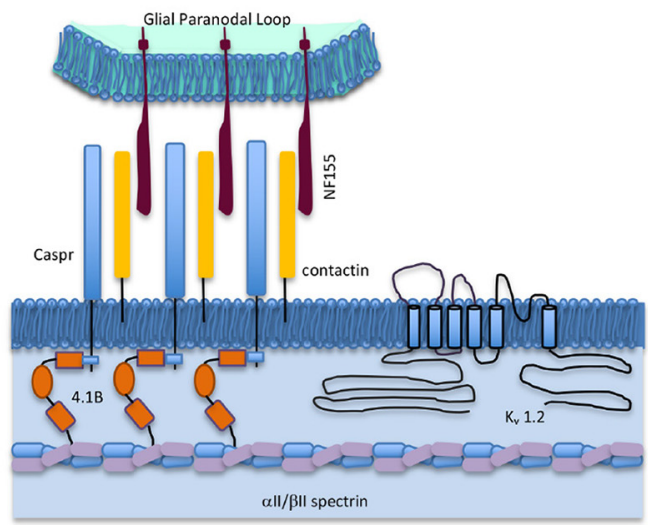

b

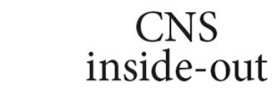

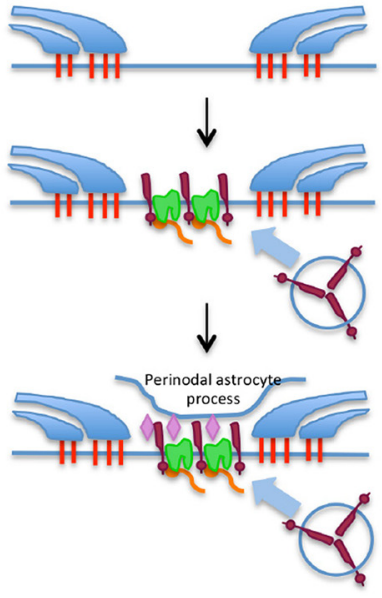

b

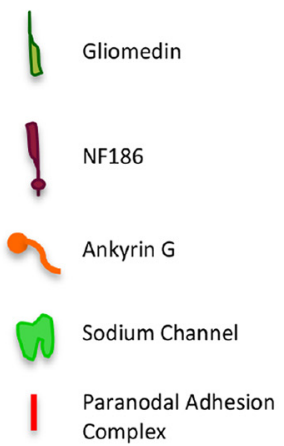

ECM

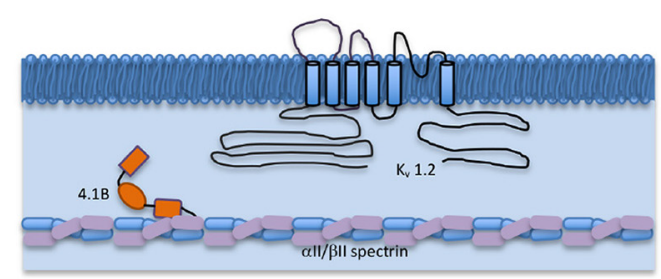

Figure 9. Schematic summary of node assembly in the PNS versus CNS. $\boldsymbol{A}$, Distinct mechanisms regulate PNS and CNS node formation. $\boldsymbol{A a}$, In the PNS, nodes assemble before the paranodes. A preexisting surface pool of NF186 (neuronal cell adhesion molecule) is concentrated by a diffusion trapping mechanism by interacting with gliomedin (glial receptor) that requires NF186 extracellular domain. It further recruits ankyrin $G$ and sodium channel to the site. This route of node formation corresponds to an "outside-in" mechanism. As nodes mature, flanked on both sides by PNJs, nodal components are replenished from intracellular pools via transport. In the case of NF186, the cytoplasmic domain is required, and mature PNJs are instrumental for this route of targeting. $\boldsymbol{A} \boldsymbol{b}$, In the CNS, paranodes form prior node formation. Nodal components assemble from intracellular pools via transport similar to the replenishment state in mature PNS node. ECM deposits around the node at a later stage and stabilizes the nodal complex. $\boldsymbol{B}$, Diffusion barrier at the paranodes. This barrier involves two sets of interaction as shown in the WT condition $(\mathbf{B a})$. One is the interaction between axolemma and paranodal glial loop, which form the extracellular membrane adhesion complex with major components of Caspr, contactin, and NF155. The second involves interactions of the adhesion complex with the submembrane cytoskeleton through 4.1B. Such interactions limit membrane proteins (e.g., Kv1.2) from entering into PNJ. $B \boldsymbol{B}$, In Caspr K0s, the membrane adhesion complex is missing and 4.1B is reduced. $\alpha\|l / \beta\|$ spectrin cytoskeleton remains intact but is not linked to the cell membrane compromising the barrier function in the paranodal region.

support here, that targeting of NF186 to mature nodes is transport-dependent. This latter pathway, exemplified by accumulation of ICAM/NF, requires trafficking, insertion, and stable expression at mature nodes. Potentially, the paranodes may determine the efficacy of each of these steps.

The paranodes are candidates to regulate local axonal transport in the nodal region and thereby nodal delivery and insertion. Thus, in normal nerves, it is known that axonal transport slows in the nodal/paranodal region, particularly in large-diameter axons, which results in local organelle accumulation (Armstrong et al., 1987; Zimmermann, 1996). In Caspr KOs, organelle accumulation at the node is further exacerbated and aberrant as evidenced by striking accumulations of abnormal appearing mitochondria (Einheber et al., 2006; Hoshi et al., 2007) and stacks of smooth endoplasmic reticulum (GarciaFresco et al., 2006). Accumulation of organelles is also seen at the nodes of paranodal cytoskeletal mutants (Saifetiarova and Bhat,
2019). This disruption of local axonal transport may result from defects in microtubule organization present at the nodes of Caspr mutants (Garcia-Fresco et al., 2006). Such defects in the Caspr mutants may likewise impair delivery of NF186-containing vesicles and of other components to the node.

In addition, defects of stability at nodes may contribute to the limited accumulation of the ICAM/NF construct at the Caspr nulls. Of note, WT NF186 is expressed normally in Caspr KO nodes in cultures and in vivo in contrast to ICAM/NF (Figs. 1C, 3 ). Yet both constructs share a common extraluminal, cytoplasmic tail, which suggests they should be transported to and inserted at nodes equivalently. Indeed, both constructs are coexpressed in the same transport vesicles (Bekku and Salzer, 2020). These results suggest that the reduction of ICAM/NF expression compared with WT NF186 may be due to relatively reduced stability. Like WT NF186, the ICAM/NF construct should bind to and be stabilized by its interactions with ankyrin $G$, which is 
robustly expressed at Caspr KO nodes (Figs. 3, 8). Limited stability could result from the lack of ectodomain interactions of the ICAM/NF construct, instability that is exacerbated by the absence of the paranode diffusion barrier. The precise contributions of defective delivery/insertion and stability that combine to limit accumulation at the node mediated by the NF186 cytoplasmic sequences remain to be established. Whether there are also broader changes in the phenotype of neurons in the Caspr KOs that also contribute to altered organization of the node beyond those described here will be of interest for future study.

\section{References}

Amor V, Zhang C, Vainshtein A, Zhang A, Zollinger DR, Eshed-Eisenbach Y, Brophy PJ, Rasband MN, Peles E (2017) The paranodal cytoskeleton clusters $\mathrm{Na}^{+}$channels at nodes of Ranvier. Elife 6:e21392.

Armstrong R, Toews AD, Morell P (1987) Axonal transport through nodes of Ranvier. Brain Res 412:196-199.

Bekku Y, Salzer JL (2020) Independent anterograde transport and retrograde cotransport of domain components of myelinated axon. J Cell Biol 219: e201906071.

Bekku Y, Rauch U, Ninomiya Y, Oohashi T (2009) Brevican distinctively assembles extracellular components at the large diameter nodes of Ranvier in the CNS. J Neurochem 108:1266-1276.

Bennett V, Lorenzo DN (2013) Spectrin- and ankyrin-based membrane domains and the evolution of vertebrates. Curr Top Membr 72:1-37.

Berger SL, Leo-Macias A, Yuen S, Khatri L, Pfennig S, Zhang Y, AgulloPascual E, Caillol G, Zhu MS, Rothenberg E, Melendez-Vasquez CV, Delmar M, Leterrier C, Salzer JL (2018) Localized myosin II activity regulates assembly and plasticity of the axon initial segment. Neuron 97:555570. e556.

Bhat MA, Rios JC, Lu Y, Garcia-Fresco GP, Ching W, St Martin M, Li J, Einheber S, Chesler M, Rosenbluth J, Salzer JL, Bellen HJ (2001) Axonglia interactions and the domain organization of myelinated axons requires neurexin IV/Caspr/Paranodin. Neuron 30:369-383.

Boguslawska DM, Machnicka B, Hryniewicz-Jankowska A, Czogalla A (2014) Spectrin and phospholipids: the current picture of their fascinating interplay. Cell Mol Biol Lett 19:158-179.

Boyle ME, Berglund EO, Murai KK, Weber L, Peles E, Ranscht B (2001) Contactin orchestrates assembly of the septate-like junctions at the paranode in myelinated peripheral nerve. Neuron 30:385-397.

Buttermore ED, Dupree JL, Cheng J, An X, Tessarollo L, Bhat MA (2011) The cytoskeletal adaptor protein band $4.1 \mathrm{~B}$ is required for the maintenance of paranodal axoglial septate junctions in myelinated axons. J Neurosci 31:8013-8024.

Charles P, Tait S, Faivre-Sarrailh C, Barbin G, Gunn-Moore F, DenisenkoNehrbass N, Guennoc AM, Girault JA, Brophy PJ, Lubetzki C (2002) Neurofascin is a glial receptor for the paranodin/Caspr-contactin axonal complex at the axoglial junction. Curr Biol 12:217-220.

Chen X, Wang Q, Ni F, Ma J (2010) Structure of the full-length Shaker potassium channel Kv1.2 by normal-mode-based X-ray crystallographic refinement. Proc Natl Acad Sci USA 107:11352-11357.

Ching W, Zanazzi G, Levinson SR, Salzer JL (1999) Clustering of neuronal sodium channels requires contact with myelinating Schwann cells. J Neurocytol 28:295-301.

D’Este E, Kamin D, Balzarotti F, Hell SW (2017) Ultrastructural anatomy of nodes of Ranvier in the peripheral nervous system as revealed by STED microscopy. Proc Natl Acad Sci USA 114:E191-E199.

Denisenko-Nehrbass N, Oguievetskaia K, Goutebroze L, Galvez T, Yamakawa H, Ohara O, Carnaud M, Girault JA (2003) Protein 4.1B associates with both Caspr/paranodin and Caspr2 at paranodes and juxtaparanodes of myelinated fibres. Eur J Neurosci 17:411-416.

Dours-Zimmermann MT, Maurer K, Rauch U, Stoffel W, Fässler R, Zimmermann DR (2009) Versican V2 assembles the extracellular matrix surrounding the nodes of Ranvier in the CNS. J Neurosci 29:7731-7742.

Dubessy AL, Mazuir E, Rappeneau Q, Ou S, Abi Ghanem C, Piquand K, Aigrot MS, Thetiot M, Desmazieres A, Chan E, Fitzgibbon M, Fleming M, Krauss R, Zalc B, Ranscht B, Lubetzki C, Sol-Foulon N (2019) Role of a Contactin multi-molecular complex secreted by oligodendrocytes in nodal protein clustering in the CNS. Glia 67:2248-2263.
Dupree JL, Girault JA, Popko B (1999) Axo-glial interactions regulate the localization of axonal paranodal proteins. J Cell Biol 147:1145-1152.

Dzhashiashvili Y, Zhang Y, Galinska J, Lam I, Grumet M, Salzer JL (2007) Nodes of Ranvier and axon initial segments are ankyrin G-dependent domains that assemble by distinct mechanisms. J Cell Biol 177:857-870.

Einheber S, Bhat MA, Salzer JL (2006) Disrupted axo-glial junctions result in accumulation of abnormal mitochondria at nodes of Ranvier. Neuron Glia Biol 2:165-174.

Einheber S, Meng X, Rubin M, Lam I, Mohandas N, An X, Shrager P, Kissil J, Maurel P, Salzer JL (2013) The 4.1B cytoskeletal protein regulates the domain organization and sheath thickness of myelinated axons. Glia 61:240-253.

Eshed Y, Feinberg K, Poliak S, Sabanay H, Sarig-Nadir O, Spiegel I, Bermingham JR Jr, Peles E (2005) Gliomedin mediates Schwann cellaxon interaction and the molecular assembly of the nodes of Ranvier. Neuron 47:215-229.

Faivre-Sarrailh C (2020) Molecular organization and function of vertebrate septate-like junctions. Biochim Biophys Acta Biomembr 1862:183211.

Faivre-Sarrailh C, Devaux JJ (2013) Neuro-glial interactions at the nodes of Ranvier: implication in health and diseases. Front Cell Neurosci 7:196.

Feinberg K, Eshed-Eisenbach Y, Frechter S, Amor V, Salomon D, Sabanay H, Dupree JL, Grumet M, Brophy PJ, Shrager P, Peles E (2010) A glial signal consisting of gliomedin and $\mathrm{NrCAM}$ clusters axonal $\mathrm{Na}^{+}$channels during the formation of nodes of Ranvier. Neuron 65:490-502.

Freeman SA, Desmazières A, Simonnet J, Gatta M, Pfeiffer F, Aigrot MS, Rappeneau Q, Guerreiro S, Michel PP, Yanagawa Y, Barbin G, Brophy PJ, Fricker D, Lubetzki C, Sol-Foulon N (2015) Acceleration of conduction velocity linked to clustering of nodal components precedes myelination. Proc Natl Acad Sci USA 112:E321-E328.

Frick M, Schmidt K, Nichols BJ (2007) Modulation of lateral diffusion in the plasma membrane by protein density. Curr Biol 17:462-467.

Garcia-Fresco GP, Sousa AD, Pillai AM, Moy SS, Crawley JN, Tessarollo L, Dupree JL, Bhat MA (2006) Disruption of axo-glial junctions causes cytoskeletal disorganization and degeneration of Purkinje neuron axons. Proc Natl Acad Sci USA 103:5137-5142.

Gollan L, Sabanay H, Poliak S, Berglund EO, Ranscht B, Peles E (2002) Retention of a cell adhesion complex at the paranodal junction requires the cytoplasmic region of Caspr. J Cell Biol 157:1247-1256.

Gollan L, Salomon D, Salzer JL, Peles E (2003) Caspr regulates the processing of contactin and inhibits its binding to neurofascin. J Cell Biol 163:12131218.

Hoover KB, Bryant PJ (2000) The genetics of the protein 4.1 family: organizers of the membrane and cytoskeleton. Curr Opin Cell Biol 12:229-234.

Horresh I, Bar V, Kissil JL, Peles E (2010) Organization of myelinated axons by Caspr and Caspr 2 requires the cytoskeletal adapter protein 4.1B. J Neurosci 30:2480-2489.

Hoshi T, Suzuki A, Hayashi S, Tohyama K, Hayashi A, Yamaguchi Y, Takeuchi K, Baba H (2007) Nodal protrusions, increased SchmidtLanterman incisures, and paranodal disorganization are characteristic features of sulfatide-deficient peripheral nerves. Glia 55:584-594.

Huang CY, Zhang C, Ho TS, Oses-Prieto J, Burlingame AL, Lalonde J, Noebels JL, Leterrier C, Rasband MN (2017) $\alpha$ II spectrin forms a periodic cytoskeleton at the axon initial segment and is required for nervous system function. J Neurosci 37:11311-11322.

Jenkins SM, Bennett V (2002) Developing nodes of Ranvier are defined by ankyrin-G clustering and are independent of paranodal axoglial adhesion. Proc Natl Acad Sci USA 99:2303-2308.

Kaplan MR, Meyer-Franke A, Lambert S, Bennett V, Duncan ID, Levinson SR, Barres BA (1997) Induction of sodium channel clustering by oligodendrocytes. Nature 386:724-728.

Kaplan MR, Cho MH, Ullian EM, Isom LL, Levinson SR, Barres BA (2001) Differential control of clustering of the sodium channels $\mathrm{Na}(\mathrm{v}) 1.2$ and $\mathrm{Na}$ (v)1.6 at developing CNS nodes of Ranvier. Neuron 30:105-119.

Lambert S, Davis JQ, Bennett V (1997) Morphogenesis of the node of Ranvier: co-clusters of ankyrin and ankyrin-binding integral proteins define early developmental intermediates. J Neurosci 17:7025-7036.

Lenne PF, Wawrezinieck L, Conchonaud F, Wurtz O, Boned A, Guo XJ, Rigneault H, He HT, Marguet D (2006) Dynamic molecular confinement in the plasma membrane by microdomains and the cytoskeleton meshwork. EMBO J 25:3245-3256.

Leterrier C (2016) The axon initial segment, 50 years later: a nexus for neuronal organization and function. Curr Top Membr 77:185-233. 
Liu CH, Stevens SR, Teliska LH, Stankewich M, Mohler PJ, Hund TJ, Rasband MN (2020) Nodal $\beta$ spectrins are required to maintain $\mathrm{Na}(+)$ channel clustering and axon integrity. Elife 9:e52378.

Lustig M, Zanazzi G, Sakurai T, Blanco C, Levinson SR, Lambert S, Grumet M, Salzer JL (2001) Nr-CAM and neurofascin interactions regulate ankyrin $\mathrm{G}$ and sodium channel clustering at the node of Ranvier. Curr Biol 11:1864-1869.

Manso C, Querol L, Lleixa C, Poncelet M, Mekaouche M, Vallat JM, Illa I, Devaux JJ (2019) Anti-Neurofascin-155 IgG4 antibodies prevent paranodal complex formation in vivo. J Clin Invest 129:2222-2236.

Maurel P, Einheber S, Galinska J, Thaker P, Lam I, Rubin MB, Scherer SS, Murakami Y, Gutmann DH, Salzer JL (2007) Nectin-like proteins mediate axon Schwann cell interactions along the internode and are essential for myelination. J Cell Biol 178:861-874.

Melendez-Vasquez CV, Rios JC, Zanazzi G, Lambert S, Bretscher A, Salzer JL (2001) Nodes of Ranvier form in association with ezrin-radixin-moesin (ERM)-positive Schwann cell processes. Proc Natl Acad Sci USA 98:1235-1240.

Nans A, Einheber S, Salzer JL, Stokes DL (2011) Electron tomography of paranodal septate-like junctions and the associated axonal and glial cytoskeletons in the central nervous system. J Neurosci Res 89:310-319.

Ogawa Y, Schafer DP, Horresh I, Bar V, Hales K, Yang Y, Susuki K, Peles E, Stankewich MC, Rasband MN (2006) Spectrins and ankyrinB constitute a specialized paranodal cytoskeleton. J Neurosci 26:5230-5239.

Oohashi T, Hirakawa S, Bekku Y, Rauch U, Zimmermann DR, Su WD, Ohtsuka A, Murakami T, Ninomiya Y (2002) Bral1, a brain-specific link protein, colocalizing with the versican V2 isoform at the nodes of Ranvier in developing and adult mouse central nervous systems. Mol Cell Neurosci 19:43-57.

Ormö M, Cubitt AB, Kallio K, Gross LA, Tsien RY, Remington SJ (1996) Crystal structure of the Aequorea victoria green fluorescent protein. Science 273:1392-1395.

Pedraza L, Huang JK, Colman DR (2001) Organizing principles of the axoglial apparatus. Neuron 30:335-344.

Perkins GA, Sosinsky GE, Ghassemzadeh S, Perez A, Jones Y, Ellisman MH (2008) Electron tomographic analysis of cytoskeletal cross-bridges in the paranodal region of the node of Ranvier in peripheral nerves. J Struct Biol 161:469-480.

Pillai AM, Thaxton C, Pribisko AL, Cheng JG, Dupree JL, Bhat MA (2009) Spatiotemporal ablation of myelinating glia-specific neurofascin (Nfasc NF155) in mice reveals gradual loss of paranodal axoglial junctions and concomitant disorganization of axonal domains. J Neurosci Res 87:17731793.

Ramadurai S, Holt A, Krasnikov V, van den Bogaart G, Killian JA, Poolman B (2009) Lateral diffusion of membrane proteins. J Am Chem Soc 131:12650-12656.

Rasband MN (2013) Cytoskeleton: axons earn their stripes. Curr Biol 23: R197-R198.

Rasband MN, Peles E (2015) The nodes of Ranvier: molecular assembly and maintenance. Cold Spring Harb Perspect Biol 8:a020495.

Rasband MN, Peles E, Trimmer JS, Levinson SR, Lux SE, Shrager P (1999) Dependence of nodal sodium channel clustering on paranodal axoglial contact in the developing CNS. J Neurosci 19:7516-7528.

Resh MD (1999) Fatty acylation of proteins: new insights into membrane targeting of myristoylated and palmitoylated proteins. Biochim Biophys Acta 1451:1-16.

Rios JC, Rubin M, St Martin M, Downey RT, Einheber S, Rosenbluth J, Levinson SR, Bhat M, Salzer JL (2003) Paranodal interactions regulate expression of sodium channel subtypes and provide a diffusion barrier for the node of Ranvier. J Neurosci 23:7001-7011.

Rosenbluth J (1976) Intramembranous particle distribution at the node of Ranvier and adjacent axolemma in myelinated axons of the frog brain. J Neurocytol 5:731-745.

Rosenbluth J (2009) Multiple functions of the paranodal junction of myelinated nerve fibers. J Neurosci Res 87:3250-3258.

Saifetiarova J, Bhat MA (2019) Ablation of cytoskeletal scaffolding proteins, Band 4.1B and Whirlin, leads to cerebellar purkinje axon pathology and motor dysfunction. J Neurosci Res 97:313-331.

Salzer JL (2003) Polarized domains of myelinated axons. Neuron 40:297-318.

Sherman DL, Tait S, Melrose S, Johnson R, Zonta B, Court FA, Macklin WB, Meek S, Smith AJ, Cottrell DF, Brophy PJ (2005) Neurofascins are required to establish axonal domains for saltatory conduction. Neuron 48:737-742.

Siggia ED, Lippincott-Schwartz J, Bekiranov S (2000) Diffusion in inhomogeneous media: theory and simulations applied to whole cell photobleach recovery. Biophys J 79:1761-1770.

Snapp EL, Altan N, Lippincott-Schwartz J (2003) Measuring protein mobility by photobleaching GFP chimeras in living cells. Curr Protoc Cell Biol Chapter 21:Unit 21.1.

Spiegel I, Adamsky K, Eshed Y, Milo R, Sabanay H, Sarig-Nadir O, Horresh I, Scherer SS, Rasband MN, Peles E (2007) A central role for Necl4 (SynCAM4) in Schwann cell-axon interaction and myelination. Nat Neurosci 10:861-869.

Susuki K, Chang KJ, Zollinger DR, Liu Y, Ogawa Y, Eshed-Eisenbach Y, Dours-Zimmermann MT, Oses-Prieto JA, Burlingame AL, Seidenbecher CI, Zimmermann DR, Oohashi T, Peles E, Rasband MN (2013) Three mechanisms assemble central nervous system nodes of Ranvier. Neuron 78:469-482.

Taylor AM, Saifetiarova J, Bhat MA (2017) Postnatal loss of neuronal and glial neurofascins differentially affects node of Ranvier maintenance and myelinated axon function. Front Cell Neurosci 11:11.

Trimble WS, Grinstein S (2015) Barriers to the free diffusion of proteins and lipids in the plasma membrane. J Cell Biol 208:259-271.

Vabnick I, Novakovic SD, Levinson SR, Schachner M, Shrager P (1996) The clustering of axonal sodium channels during development of the peripheral nervous system. J Neurosci 16:4914-4922.

Xu K, Zhong G, Zhuang X (2013) Actin, spectrin, and associated proteins form a periodic cytoskeletal structure in axons. Science 339:452-456.

Yageta M, Kuramochi M, Masuda M, Fukami T, Fukuhara H, Maruyama T, Shibuya M, Murakami Y (2002) Direct association of TSLC1 and DAL-1, two distinct tumor suppressor proteins in lung cancer. Cancer Res 62:5129-5133.

Zhang C, Susuki K, Zollinger DR, Dupree JL, Rasband MN (2013) Membrane domain organization of myelinated axons requires $\beta$ II spectrin. J Cell Biol 203:437-443.

Zhang Y, Bekku Y, Dzhashiashvili Y, Armenti S, Meng X, Sasaki Y, Milbrandt J, Salzer JL (2012) Assembly and maintenance of nodes of Ranvier rely on distinct sources of proteins and targeting mechanisms. Neuron 73:92-107.

Zimmermann H (1996) Accumulation of synaptic vesicle proteins and cytoskeletal specializations at the peripheral node of Ranvier. Microsc Res Tech 34:462-473.

Zonta B, Tait S, Melrose S, Anderson H, Harroch S, Higginson J, Sherman DL, Brophy PJ (2008) Glial and neuronal isoforms of Neurofascin have distinct roles in the assembly of nodes of Ranvier in the central nervous system. J Cell Biol 181:1169-1177. 